\title{
Problema de Riemann para Leis de Conservação Escalares
}

\author{
Ana Paula VitTi Barcha
}

Orientador: Prof. Dr. Wagner Vieira Leite Nunes

Dissertação apresentada ao Instituto de Ciências Matemáticas de São Carlos-USP, como parte dos requisitos para obtenção do título de "Mestre em Ciências - Área: Matemática”.

\section{USP - São Carlos}

1995 
Mas aos que esperam no Senhor renovarão as suas forças, subirão com asas como águias: correrão, e não se cansarão: caminharão, e não se fatigarão. Isaias 40:31 
Aos meus pais, a minha irmã e meu cunhado e ao meu marido, pelo apoio e pelas horas de convivência roubadas 


\section{Agradecimentos}

Ao professor Wagner Vieira Leite Nunes pela orientação, dedicação, paciência e amizade no decorrer do desenvolvimento deste trabalho.

Ao amigo Marcos que sempre me ajudou nas horas mais difíceis.

Às secretárias Laura e Beth pela simpatia, atenção e eficiência com que sempre me atenderam.

Aos meus amigos que me proporcionaram momentos alegres e que me deram apoio em momentos difíceis neste período, em especial: Vera e Neyva.

Ao Conselho Nacional de Desenvolvimento Científico e Tecnológico CNPq, pela concessão de uma bolsa de estudos para desenvolvimento de programa de mestrado junto a área de Pós-Graduação em matemática pura do ICMSC-USP.

E, agradeço acima de tudo á Deus que sempre me iluminou, mostrando-me os caminhos a serem seguidos a fim de superar os obstáculos e conquistar os meus objetivos. 


\section{RESUMO}

Este trabalho apresenta um estudo sobre a existência, unicidade e comportamento assintótico de soluções para uma lei de conservação escalar da forma $u_{t}+f(u)_{x}=$ $=0$. Esta solução satisfaz a desigualdade de entropia, e além disso é a única solução com esta propriedade. O problema é estudado via o método das diferenças finitas. 


\begin{abstract}
In this work we studied the existence, uniqueness and asymptotic behavior of solutions for a single conservation law in the form $u_{t}+f(u)_{x}=0$. This solution satisfies the entropy inequality, and furthermore is the unique solution with this propriety. The problem was studied using the finite-difference method.
\end{abstract}




\section{Índice}

$\begin{array}{ll}\text { Introdução } & 1\end{array}$

0.1 Considerações iniciais . . . . . . . . . . . . . . . . . . 1

0.2 Objetivo deste Trabalho . . . . . . . . . . . . . . . 1

0.3 Conteúdo do Trabalho . . . . . . . . . . . . . . 2

1 Preliminares $\quad 3$

1.1 Funções de Variação Limitada . . . . . . . . . . . . . . . . . . 3

1.2 Princípio da Escolha de Helly . . . . . . . . . . . . . . . 4

2 Soluções Descontínuas de Leis de Conservação 9

2.1 Soluções Descontínuas . . . . . . . . . . . . . . . . 9

2.2 Soluções Fracas de Leis de Conservação . . . . . . . . . . . . . . . . . 11

$2.3 \quad$ Irreversibilidade . . . . . . . . . . . . . . . . . . 20

3 Leis de conservação escalar $\quad 22$

3.1 Existência de uma solução de entropia . . . . . . . . . . . . . 22

3.2 Unicidade da solução de entropia . . . . . . . . . . . . . . . 41

3.3 Comportamento assintótico das soluções de entropia . . . . . . . . . 50

Bibliografia $\quad 59$ 


\section{Introdução}

\subsection{Considerações iniciais}

A teoria matemática de leis de conservação data de 1950, conforme o trabalho de E. Hopf, e foi seguido por uma série de outros trabalhos entre os quais destacamos de $\mathrm{O}$. Oleinik [7] juntamente com Lax [5]. A teoria de soluções descontínuas ficou parada até o surgimento da noção de soluções fracas e distribuições se tornarem fami-liares. Naturalmente muitos trabalhos importantes foram realizados a priori através de dinâmica de fuidos começando no século XIX.

Durante a segunda guerra mundial, a invenção de aviões capazes de superar a velocidade do som gerou a necessidade de uma teoria que melhor entendesse o fenômeno de choque e das equações de dinâmica de gases. Muitos matemáticos "puros" encantaramse por este estudo, entre outros mencionamos J.von Neumann e H. Weyl. Em meados de 1950 fenômenos de ondas não lineares atraíram a atenção de físicos teóricos tais como Fermi, Pasta e Ulam.

\subsection{Objetivo deste Trabalho}

Neste trabalho obteremos resultados matemáticos precisos sobre existência e unicidade de soluções para uma Lei de conservação escalar. Estudaremos também o comportamento assintótico da solução . O problema existente será abordado via o método das diferenças finitas, que apareceu primeiramente num trabalho de Lax, [4]. Assim substituiremos a equação diferencial dada por uma aproximação de diferenças finitas, dependendo dos parâmetros de malha $\Delta x$ e $\Delta t$. Para cada par $(\Delta x, \Delta t)$ construiremos uma solução $U_{\Delta x, \Delta t}$ de equações de diferenças finitas e então obteremos estimativas que nos permitirá passar o limite com o parâmetro de malha tendendo para zero num certo caminho definido. A estimativa obtida será na norma do sup e na norma da variação total. O teorema de existência e unicidade que queremos mostrar é devido a Olienik [7], sendo que a prova aqui apresentada será uma adaptação deste. 
Existem mais quatro outras aproximações matemáticas diferentes para o problema de Leis de conservação escalar existente. São elas: (i) cálculo de variações e Teoria de Hamilton-Jacobi; (ii) o método da viscosidade; (iii) teoria de semi-grupos não lineares; e (iv) o método das características. Todos estes métodos são muito interessantes. Contudo decidimos fazer a prova via diferenças finitas devido ao fato de serem mais facilmente generalizadas para o caso de sistemas de leis de conservação. Mostraremos que a solução satisfaz a desigualdade de entropia e além disso que é a única com esta propriedade. A desigualdede de entropia também será usada para estudar o comportamento assintótico das soluções com $t$ tendendo para infinito. Mostraremos também que se o dado inicial tem suporte compacto a solução decai em $L^{1}(-R, R)$. Para este tipo particular de solução damos o nome de $\mathrm{N}$ - onda. Também estudaremos o comportamento de soluções com dado inicial periódico.

\subsection{Conteúdo do Trabalho}

Esta trabalho é composto pelos seguintes capítulos:

\section{CAPÍTULO 1 - PRELIMINARES}

Este capítulo apresenta o estudo de noções preliminares usadas nos posteriores.

\section{CAPÍTULO 2 - SOLUÇÕES DESCONTÍNUAS DE LEIS DE CONSERVA ÇÃO}

Neste capítulo encontra-se o estudo de sistemas quase - lineares da forma $u_{t}+$ $f(u)_{x}=0$ onde $u \in \mathbb{R}$ e consideraremos alguns exemplos e descreveremos certos fenômenos que ocorrem.

\section{CAPÍTULO 3 - LEIS DE CONSERVAÇÃO ESCALAR}

O teorema de existência de unicidade de soluções para uma lei de conservação escalar é enunciado e demonstrado neste capítulo e também apresentamos o estudo do comportamento assintótico da solução. O problema de existêcia será resolvido via o método das diferenças finitas. 


\section{Capítulo 1}

\section{Preliminares}

Neste capítulo vamos dar alguns conceitos que serão utilizados nos capítulos posteriores.

\subsection{Funções de Variação Limitada}

Nesta secção discutiremos a teoria de uma importante classe de funções que são as funções de variação limitada.

Seja $f(x)$ uma função definida sobre o intervalo $[a, b]$ e

$$
P: a=x_{0}<x_{1}<x_{2}<\cdots<x_{n}=b
$$

uma partição de $[a, b]$ e consideremos a soma

$$
V_{p}=\sum_{k=0}^{n-1}\left|f\left(x_{k+1}\right)-f\left(x_{k}\right)\right|
$$

Definição 1.1.1 O menor limite superior do conjunto de todas as possíveis somas $V_{p} e ́$ chamado a variação total da função $f(x)$ em $[a, b]$ e será denotado por $V_{a}^{b}(f)=i n f_{p} V_{p}$. Se

$$
V_{a}^{b}(f)<+\infty
$$

então dizemos que $f(x)$ é uma função de variação limitada sobre $[a, b]$. Também dizemos que $f(x)$ tem variação limitada sobre $[a, b]$. 
Teorema 1.1.1 Uma função $f(x)$ definida e limitada sobre $[a, b]$ é uma função de variação limitada se, e somente se, é representada como a diferença de duas funções crescentes.

\subsection{Princípio da Escolha de Helly}

Nesta secção falaremos de um teorema devido a E. Helly. Primeiro daremos dois lemas.

Lema 1.2.1 Seja uma família infinita de funções $H=\{f(x)\}$ definida sobre $[a, b]$. Se todas as funções da família são limitadas digamos

$$
|f(x)| \leq k
$$

então para qualquer subconjunto enumerável $E$ de $[a, b]$ é possivel encontrar uma sequência $\left\{f_{n}(x)\right\}$ na família $H$ que converge para todo ponto do conjunto $E$.

Prova: Seja $E=\left\{x_{k}\right\}$. Consideremos o conjunto

\section{$\left\{f_{1}\right\}$}

de valores obtidos pelas funções da família $H$ no ponto $x_{1}$. Por (1.1) temos que este conjunto é limitado, e pelo teorema de Bolzano- Weierstrass, podemos selecionar uma sequência convergente

$$
f_{1}^{(1)}\left(x_{1}\right), f_{2}^{(1)}\left(x_{1}\right), f_{3}^{(1)}\left(x_{1}\right), \ldots ; \lim _{n \rightarrow \text { infty }} f_{n}^{(1)}\left(x_{1}\right)=A_{1}
$$

Agora considere a sequência

$$
f_{1}^{(1)}\left(x_{2}\right), f_{2}^{(1)}\left(x_{2}\right), f_{3}^{(1)}\left(x_{2}\right), \ldots
$$

de valores obtidos pelas funções do conjunto $\left\{f_{n}^{(1)}(x)\right\}$ no ponto $x_{2}$. Esta sequência é também limitada, e podemos a plicar o teorema de Bolzano-Weierstrass para ela. Isto nos dá uma sequência convergente 


$$
f_{1}^{(2)}\left(x_{2}\right), f_{2}^{(2)}\left(x_{2}\right), f_{3}^{(2)}\left(x_{2}\right), \ldots ; \lim _{n \rightarrow \infty} f_{n}^{(2)}\left(x_{2}\right)=A_{2}
$$

selecionada de $\left\{f_{n}^{(1)}\left(x_{2}\right)\right\}$. É essencial notar que a ordem relativa das funções $f_{n}^{(2)}$ e $f_{m}^{(2)}$ em (1.3) é a mesma que em (1.2). Continuando este processo indefinidamente, construímos uma sequência convergente

$$
\begin{aligned}
& f_{1}^{(1)}\left(x_{1}\right), f_{2}^{(1)}\left(x_{1}\right), f_{3}^{(1)}\left(x_{1}\right), \ldots ; \lim _{n \rightarrow \infty} f_{n}^{(1)}\left(x_{1}\right)=A_{1} \\
& f_{1}^{(2)}\left(x_{2}\right), f_{2}^{(2)}\left(x_{2}\right), f_{3}^{(2)}\left(x_{2}\right), \ldots ; \lim _{n \rightarrow \infty} f_{n}^{(2)}\left(x_{2}\right)=A_{2} \\
& \ldots \ldots \ldots \ldots \ldots \ldots \ldots \ldots \ldots \ldots \ldots \ldots \ldots \ldots \ldots \\
& f_{1}^{(k)}\left(x_{k}\right), f_{2}^{(k)}\left(x_{k}\right), f_{3}^{(k)}\left(x_{k}\right), \ldots ; \lim _{n \rightarrow \infty} f_{n}^{(k)}\left(x_{k}\right)=A_{k}
\end{aligned}
$$

onde cada sequência é uma subsequência da anterior e para a qual a ordem dos elementos não foi alterada.

Podemos formar a sequência de elementos diagonais da matriz infinita construida, isto é, a sequência

$$
\left\{f_{n}^{(n)}(x)\right\} \quad(n=1,2,3 \ldots)
$$

esta sequência converge em todos os pontos do conjunto $E$. De fato para todo $k$ fixado a sequência

$$
\left\{f_{n}^{(n)}\left(x_{k}\right)\right\} \quad(n \geq k)
$$

é uma subsequência de $\left\{f_{n}^{(k)}\left(x_{k}\right)\right\}$ e converge para $A_{k}$.

Lema 1.2.2 Seja $F=\{f(x)\}$ uma família infinita de funções crescentes definida sobre o segmento $[a, b]$. Se todas as funções da família são limitadas digamos

$$
|f(x)| \leq k, \quad f \in F, \quad a \leq x \leq b
$$

então existe uma sequência de funções $\left\{f_{n}(x)\right\}$ em $F$ que converge para uma função crescente $\phi(x)$ em todos os pontos de $[a, b]$. 
Prova: Aplicamos o lema (1.2.1) para $\{f(x)\}$, obtendo para $E$ o conjunto consistindo de todos os pontos racionais de $[a, b] a$ podendo ser irracional. Assim encontramos uma sequência de funções da família $F$,

$$
\lim _{n \rightarrow \infty} f^{(n)}\left(x_{k}\right)
$$

tal que

$$
F_{0}=\left\{f^{(n)}(x)\right\}
$$

existe e é finita para todo ponto $x_{k} \in E$.

Vamos definir agora uma função $\phi(x)$ pelo seguinte procedimento. Primeiro definimos

$$
\phi\left(x_{k}\right)=\lim _{n \rightarrow \infty} f^{(n)}\left(x_{k}\right) \quad\left(x_{k} \in E\right)
$$

para todo ponto $x_{k} \in E$. Isto define $\phi(x)$ somente sobre $E$, naturalmente. Podemos ver que $\phi(x)$ é uma função decrescente sobre $E$, isto é, se $x_{i}, x_{k} \in E$ e $x_{i}<x_{k}$ então

$$
\phi\left(x_{k}\right)<\phi\left(x_{i}\right)
$$

para $x \in[a, b]-E$, definimos $\phi(x)$ pela relação

$$
\phi(x)=\sup _{x_{k}<\infty}\left\{\phi\left(x_{k}\right)\right\} \quad\left(x_{k} \in E\right)
$$

Claramente vemos que $\phi(x)$ é uma função decrescente sobre o intervalo $[a, b]$ e que o conjunto de pontos $Q$ onde $\phi(x)$ é descontínua é quando muito não enumerável.

Mostraremos agora que

$$
\lim _{n \rightarrow \infty} f^{(n)}\left(x_{0}\right)=\phi\left(x_{0}\right)
$$

em todo ponto $x_{0}$ onde $\phi(x)$ é contínua. Seja $\varepsilon>0$ e sejam $x_{k}$ e $x_{i}$ pontos de $E$ tal que

$$
x_{k}<x_{0}<x_{i}, \quad \phi\left(x_{i}\right)-\phi\left(x_{k}\right)<\frac{\varepsilon}{2}
$$

Fixando os pontos $x_{k}$ e $x_{i}$ tomemos $n_{0}$ tal que $n>n_{0}$

$$
\left|f^{(n)}\left(x_{k}\right)-\phi\left(x_{k}\right)\right|<\frac{\varepsilon}{2} \quad\left|f^{(n)}\left(x_{i}\right)-\phi\left(x_{i}\right)\right|<\frac{\varepsilon}{2}
$$


Podemos ver que

$$
\phi\left(x_{0}\right)-\varepsilon<f^{(n)}\left(x_{k}\right) \leq f^{(n)}\left(x_{i}\right)<\phi\left(x_{0}\right)+\varepsilon
$$

para $n>n_{0}$. Como

$$
f^{(n)}\left(x_{k}\right) \leq f^{(n)}\left(x_{0}\right) \leq f^{(n)}\left(x_{i}\right)
$$

temos

$$
\phi\left(x_{0}\right)-\varepsilon<f^{(n)}\left(x_{0}\right)<\phi\left(x_{0}\right)+\varepsilon
$$

para $n>n_{0}$. Isto prova (1.5). Assim a igualdade

$$
\lim _{n \rightarrow \infty} f^{(n)}(x)=\phi(x)
$$

pode falhar somente sobre o conjunto finito ou não enumerável $Q$ onde $\phi(x)$ for descontínua.

Aplicamos agora o lema (1.2.1) para a sequência $F_{0}$ tomando para o conjunto $E$ o conjunto daqueles pontos de $Q$ onde (1.6) não está satisfeita. Isto nos dá uma subsequência $\left\{f_{n}(x)\right\}$ de $F_{0}$ que converge em todos os pontos de $[a, b]$. Colocando

$$
\phi(x)=\lim _{n \rightarrow \infty} f_{n}(x)
$$

com isto obtemos uma função decrescente.

Teorema 1.2.1 (Teorema de Helly). Seja uma família infinita de funções $F=\{f(x)\}$ definida sobre o segmento $[a, b]$. Se todas as funções da família e suas variações totais são limitadas por um mesmo número

$$
|f(x)| \leq k, \quad V_{a}^{b}(f) \leq k
$$

então existe uma sequência $\left\{f_{n}(x)\right\}$ na família $F$ que converge em cada ponto de $[a, b]$ para alguma função $\phi(x)$ de variação total limitada.

Prova: Para toda função $f(x)$ da família $F$, seja

$$
\pi(x)=V_{a}^{x}(f), \quad \nu(x)=\pi(x)-f(x)
$$


vemos que $\pi(x)$ e $\mu(x)$ são funções crescentes. Além disso,

$$
|\pi(x)| \leq k, \quad|\mu(x)| \leq 2 k
$$

aplicando o lema (1.2.2) para a família $\{\pi(x)\}$, existe uma sequência convergente $\left\{\pi_{k}(x)\right\}$,

$$
\lim _{k \rightarrow \infty} \pi_{k}(x)=\alpha(x)
$$

nesta família. Para toda função $\pi_{k}(x)$ existe uma correspondente função $\mu_{k}(x)$, que se extende para a função $f_{k}(x)$ da família $F$. Aplicando o lema (1.2.2) para a família $\left\{\mu_{k}(x)\right\}$ encontramos uma subsequência convergente $\left\{\mu_{k_{i}}\right\}$,

$$
\lim _{i \rightarrow \infty} \mu_{k_{i}}(x)=\beta(x)
$$

de $\left\{v_{k}(x)\right\}$. Então a sequência de funções

$$
f_{k_{i}}(x)=\pi_{k_{i}}(x)-\mu_{k_{i}}(x)
$$

pertence a $F$, e convergem para a função

$$
\phi(x)=\alpha(x)-\beta(x)
$$

Isto prova o teorema de Helly. 


\section{Capítulo 2}

\section{Soluções Descontínuas de Leis de Conservação}

Neste capítulo começaremos o estudo de sistemas quase- lineares da forma

$$
u_{t}+f(u)_{x}=0
$$

onde $u=\left(u_{1}, \cdots, u_{n}\right) \in \mathbb{R}^{n}, n \geq 1,(x, t) \in \mathbb{R} \times \mathbb{R}_{+}$e $f \in C^{2}$ em algum subconjunto $\Omega \in \mathbb{R}^{n}$. Esta equação é chamada de lei de conservação . Consideraremos alguns exemplos e descreveremos certos fenômenos, associados a estes.

Observação 2.0.1 O sistema (2.1) surgiu a partir do estudo de fenômenos de ondas não lineares. Este sistema admite soluções descontínuas, isto é, ondas de choque.

\subsection{Soluções Descontínuas}

Considere a equação escalar, chamada de equação de Burgers,

$$
u_{t}+\left(\frac{u^{2}}{2}\right)_{x}=0
$$

a qual podemos escrever na forma:

$$
u_{t}+u u_{x}=0
$$

Esta equação tem a propriedade de ter soluções somente $C^{1}$ para o qual estão satisfeitas em $t>0$. São monotonicamente não decrescentes em $x$ para cada $t>0$ fixado. 
Suponhamos que $u$ é uma solução $C^{1}$ em $t>0$. Para qualquer ponto $\left(x_{0}, t_{0}\right), t_{0}>0$ consideremos a única solução da curva $x(t)$ que resolve

$$
\frac{d x}{d t}=u(x, t), \quad x\left(t_{0}\right)=x_{0} .
$$

ao longo desta curva que é uma característica de (2.3),

$$
\frac{d}{d t} u(x(t), t)=u_{x} \frac{d x}{d t}+u_{t}=u_{x} u+u_{t}=0 .
$$

Assim $u$ é constante ao longo das características e de (2.4) podemos concluir que as características são retas tendo velocidade igual ao valor de $u$ ao longo dessas retas. E isto implica que para cada $t \geq 0, u\left(x_{1}, t\right) \leq u\left(x_{2}, t\right)$ se $x_{1}<x_{2}$ pois caso contrário as retas características deveriam se encontrar em algum ponto em $t>0$ mas isto não pode acontecer já que $u$ é suave em $t>0$.

Assim se $u(x, 0)=u_{0}(x)$ e $u_{0}^{\prime}(\bar{x})<$ $<0$ para algum $\bar{x}$ então (2.2) não pode ter solução definida em todo $t>0$. Para entender me-lhor, vamos considerar a função $u_{0} \in C^{\infty}$ cujo gráfico é descrito na figura 2.1.

Aqui $u_{0}(x)=1$ se $x \leq 0, u_{0}(x)=0$ se $x \geq 1$ e $u_{0}^{\prime} \leq 0$. Podemos esboçar as características na figura 2.2 .

Considere a região $0 \leq t \leq 1$. Na região $A$ a característica tem inclinação 1 e a solução tem o valor constante 1; na região $C$ a característica tem inclinação infinita e a solução tem valor constante 0 . Mas na região $B$ vemos que $u$ decresce de 1 para 0 quando $t$ cresce.

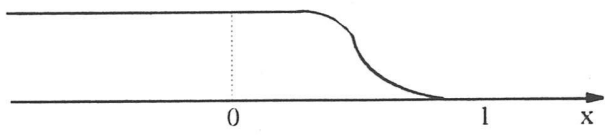

Figura 2.1

Mais geralmente, considere o problema de valor inicial para $u$

$$
u_{t}+f(u)_{x}=0, \quad t>0 ; \quad u(x, 0)=u_{0}(x), \quad x \in \mathbb{R} .
$$


Podemos reescrever esta equação como $u_{t}+f^{\prime}(u) u_{x}=0$ e considerarmos as características

$$
\frac{d t}{d s}=1, \quad \frac{d x}{d s}=f^{\prime}(u)
$$

ao longo de uma tal curva,

$$
\frac{d u}{d s}=\frac{d t}{d s} u_{t}+\frac{d x}{d s} u_{x}=u_{t}+f^{\prime}(u) u_{x}=0
$$

Assim $u$ é constante ao - longo das características. Como a inclinação das características é $1 / f^{\prime}(u)$ então elas são retas tendo inclinação determinada por seus valores em $t=0$, isto é, por $u_{0}(x)$. Então se existem pontos $x_{1}<x_{2}$ com

$$
m_{1}=\frac{1}{f^{\prime}\left(u_{0}\left(x_{1}\right)\right)}<\frac{1}{f^{\prime}\left(u_{0}\left(x_{2}\right)\right)}=m_{2}
$$

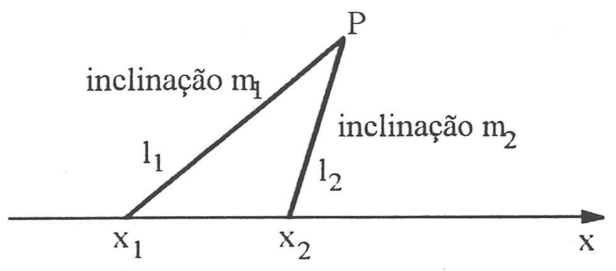

Figura 2.3

então as caraccterísticas começando em $\left(x_{1}, 0\right)$ e $\left(x_{2}, 0\right)$ se cruzarão em $t>0$. Veja Figura 2.3

Ao longo de $l_{i}, u(x, t) \equiv u_{0}\left(x_{i}\right), i=1,2$. Assim em $P$ a solução pode ser descontínua. Note que esta conclusão é independente da propriedade de suavidade de $f$ e $u_{0}$. Elas podem ser analítica e ainda assim podemos obter uma solução não definida globalmente.

Observação 2.1.1 Se $f^{\prime}\left(u_{0}\left(x_{i}\right)\right)>0$ as características não se cruzam e se $f^{\prime}\left(u_{0}\left(x_{i}\right)\right)<$ $<0$ as características se cruzam.

\subsection{Soluções Fracas de Leis de Conservação}

Nesta secção generalizaremos a noção de solução para a equação da forma (2.1). Assim consideremos o problema de valor inicial para (2.1) em $t>0$

$$
u_{t}+f(u)_{x}=0, \quad u(x, 0)=u_{0}(x) .
$$


Vamos supor por um momento que $u$ é solução clássica de (2.6). Seja $\phi \in C^{1}$ tal que $\phi \equiv 0$ fora de um conjunto compacto em $t \geq 0$, isto é, $(\operatorname{spt} \phi) \cap$ $(t \geq 0) \subseteq D$, onde $D$ é o retângulo $0 \leq t \leq T, a \leq x \leq b$ e também escolhemos $\phi \equiv 0$ fora de $D$ e sobre a reta $t=T, x=a$ e $x=b$, veja Figura

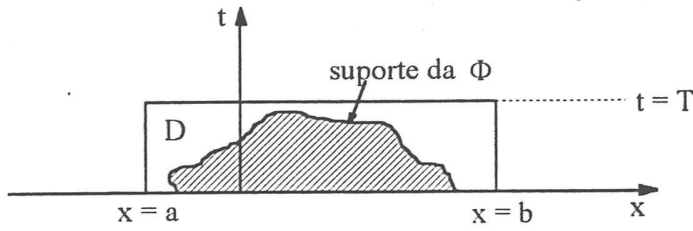

Figura 2.4

2.4

Se multiplicarmos (2.6) por $\phi$ e integrarmos sobre $t$ conseguimos

$$
\iint_{t>0}\left(u_{t}+f_{x}\right) \phi d x d t=\iint_{D}\left(u_{t}+f_{x}\right) \phi d x d t=\int_{a}^{b} \int_{0}^{T}\left(u_{t}+f_{x}\right) \phi d x d t=0 .
$$

Agora integrando por partes temos

$$
\begin{aligned}
\int_{a}^{b} \int_{0}^{T} u_{t} \phi d x d t & =\left.\int_{a}^{b} u \phi\right|_{t=0} ^{t=T} d x-\int_{a}^{b} \int_{0}^{T} u \phi_{t} d x d t \\
& =\int_{a}^{b}-u_{0}(x) \phi(x, 0) d x-\int_{a}^{b} \int_{0}^{T} u \phi_{t} d x d t
\end{aligned}
$$

e

$$
\int_{0}^{T} \int_{a}^{b} f_{x} \phi d x d t=\left.\int_{0}^{T} f \phi\right|_{x=a} ^{x=b} d t-\int_{0}^{T} \int_{a}^{b} f \phi_{x} d x d t
$$

Assim finalmente, obtemos

$$
\iint_{t \geq 0}\left(u \phi_{t}+f(u) \phi_{x}\right) d x d t+\int_{t=0} u_{0} \phi d x=0 .
$$

Mostramos que se u é uma solução clássica de (2.6), então (2.7) acontece para todo $\phi \in C_{0}^{1}$. Mas (2.7) faz sentido se $u$ e $u_{0}$ são meramente limitadas e mensuráveis.

Definição 2.2.1 Uma função mensurável limitada $u(x, t)$ é chamada solução fraca do problema de valor inicial (2.6) com dado inicial $u_{0}$, limitado e mensurável, contanto que (2.7) aconteça para toda $\phi \in C_{0}^{1}$. 
Note que (2.7) vale para toda $\phi \in C_{0}^{1}$ então se $u$ é $C^{1}$, $u$ é uma solução classica de (2.6). Isto é fácil de ver pois se $\phi$ tem suporte em $t>0$ então podemos integrar. (2.7) por partes e conseguir para toda tal $\phi$

$$
\iint_{t>0}\left(u_{t}+f_{x}\right) \phi=0
$$

tal que $u_{t}+f(u)_{x}=0$. Se agora $\phi \in C_{0}^{1}$ podemos multiplicar esta última equação por $\phi$ e integrar por partes sobre $D$ e obtemos

$$
\iint_{t>0}\left(u \phi_{t}+f \cdot \phi_{x}\right) d x d t+\int_{t=0} u(x, 0) \phi(x, 0) d x=0
$$

Comparando esta com (2.7) temos

$$
\int_{t=0}\left(u(x, 0)-u_{0}(x)\right) \phi(x, 0) d x=0
$$

e como $u_{0}$ é contínua a arbitrariedade de $\phi$ implica $u(x, 0)=u_{0}(x)$. Assim, temos mostrado que o conceito de solução dado pela definição (2.2.1) é uma generalização da noção de solução clássica.

Mostraremos agora que nenhuma descontinuidade é permitida, de fato, a condição (2.7) coloca severas restrições nas curvas de descontinuidade.

Para este fim seja $\Gamma$ uma curva suave através da qual $u$ tem um salto de descontinuidade, isto é, $u$ tem limite definido em ambos os lados de $\Gamma$ e $u$ é um caminho suave de $\Gamma$. Seja $P$ qualquer ponto de $\Gamma$ e seja $D$ a menor bola centrada em $P$. Assumimos que em $D, \Gamma$ é dada por $x=x(t)$. Sejam $D_{1}$ e $D_{2}$ as componentes de $D$ que são determinadas por $\Gamma$. Veja Figura 2.5.

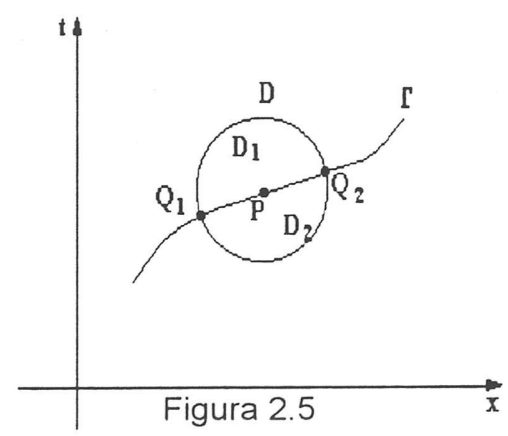

Seja $\phi \in C_{0}^{1}(D)$ então de $(2.7)$ temos

$$
0=\iint_{D}\left(u \phi_{t}+f \phi_{x}\right) d x d t=\iint_{D_{1}}\left(u \phi_{t}+f \phi_{x}\right) d x d t+\iint_{D_{2}}\left(u \phi_{t}+f \phi_{x}\right) d x d t
$$

Usando o fato que $u$ é $C^{1}$ em $D_{i}$ temos do teorema da divergência que

$$
\iint_{D_{i}}\left(u \phi_{t}+f \phi_{x}\right) d x d t=\iint_{D_{i}}(u \phi)_{t}+(f \phi)_{x}=\int_{\partial D_{i}} \phi(-u d x+f d t)
$$


como $\phi=0$ em $\partial D$ esta integral de linha é diferente de zero somente ao longo de $\Gamma$. Assim se $u_{l}=u(x(t)-0, t)$ e $u_{r}=u(x(t)+0, t)$ então temos

$$
\begin{gathered}
\int_{\partial D_{1}} \phi(-u d x+f d t)=\int_{Q_{1}}^{Q_{2}} \phi\left(-u_{l} d x+f\left(u_{l}\right) d t\right) \\
\int_{\partial D_{2}} \phi(-u d x+f d t)=-\int_{Q_{1}}^{Q_{2}} \phi\left(-u_{r} d x+f\left(u_{r}\right) d t\right)
\end{gathered}
$$

portanto

$$
0=\int_{\Gamma} \phi(-[u] d x+[f(u)] d t)
$$

onde, $[u]=u_{l}-u_{r}$ é o salto através de $\gamma$ e similarmente $[f(u)]=f\left(u_{l}\right)-f\left(u_{r}\right)$. Como $\phi$ era arbitrário concluímos que

$$
s[u]=[f(u)]
$$

em cada ponto de $\Gamma$ onde $s=\frac{d x}{d t}$, pois

$$
[u] \frac{d x}{d t}=\frac{d}{d t} \int_{\Gamma}[u] d x=\frac{d}{d t} \int_{\Gamma}[f(u)] d t=[f(u)]
$$

Chamaremos $s$ de velocidade de descontinuidade. Temos assim mostrado que (2.7) implica a relação (2.8), uma equação que liga o valor de $u$ sobre ambos os lados da curva de descontinuidade com $f$ e a velocidade de descontinuidade. A relação (2.8) é chamada de condição de salto, em dinâmica de gas é conhecida como a condição de "Rankine-Hugoniot".

Exemplo 2.2.1 Se considerarmos a equação de Burgers (2.D) então (2.8) implica

$$
s\left(u_{l}-u_{r}\right)=\frac{u_{l}^{2}}{2}-\frac{u_{r}^{2}}{2}=\frac{1}{2}\left(u_{l}^{2}-u_{r}^{2}\right)
$$

Logo, como $u_{l} \neq u_{r}, s=\left(u_{l}+u_{r}\right) / 2$. Assim a velocidade de descontinuidade é a média do valor limitante de ambos os lados da descontinuidade. Podemos usar esta relação para resolver explicitamente o problema de valor inicial e assim obter uma solução que não é clássica. 
Consideremos a equação de Burgers com dado inicial

$$
u(x, 0)=\left\{\begin{array}{lc}
1, & x<0, \\
1-x, & 0 \leq x \leq 1, \\
0, & x>1 .
\end{array}\right.
$$

observemos que as características são

$$
x=b\left(u_{0}\left(x_{0}\right)\right) t+x_{0}=u_{0}\left(x_{0}\right) t+x_{0}
$$

se $x_{0}<0 \Rightarrow u_{0}\left(x_{0}\right)=1 \Rightarrow x=t+x_{0}$.

Se $0 \leq x_{0} \leq 1 \Rightarrow u_{0}\left(x_{0}\right)=1-x_{0} \Rightarrow x=\left(1-x_{0}\right) t+x_{0}$.

Se $x_{0}>1 \Rightarrow u_{0}\left(x_{0}\right)=0 \Rightarrow x=x_{0}$.

$\log \mathrm{O}$

$$
x=\left\{\begin{array}{lc}
t+x_{0}, & x_{0}<0, \\
\left(1-x_{0}\right) t+x_{0}, & 0 \leq x_{0} \leq 1, \\
x=x_{0}, & x_{0}>1 .
\end{array}\right.
$$

há cruzamento de características, com isto só podemos tentar resolver o problema para $0 \leq t<1$. Se $x<t<1 \Rightarrow \exists x_{0}<0$ tal que $x=t+x_{0} \Rightarrow u(x, t)=u_{0}\left(x_{0}\right)=1$.

Se $t<x<1 \Rightarrow \exists 0 \leq x_{0} \leq 1$ tal que $x=\left(1-x_{0}\right) t+x_{0} \Rightarrow u(x, t)=\frac{1-x}{1-t}$.

Se $t<1<x \Rightarrow \exists x_{0}>1$ tal que $x=x_{0} \Rightarrow u(x, t)=u_{0}\left(x_{0}\right)=0$.

$\log$ temos que

$$
u(x, t)=\left\{\begin{array}{lr}
1, & x<t<1, \\
\frac{1-x}{1-t}, & t<x<1, \\
0, & t<1<x .
\end{array}\right.
$$


vemos que isto é definido somente para $t<1$. Para $t \geq 1$ usamos (2.7) $\operatorname{com} u_{l}=1$, $u_{r}=0$ tomemos $s=\frac{1}{2}$ e obtemos $x(t)=t / 2+c$.

Por outro lado a descontinuidade deve começar no ponto $(1,1)$. Logo a curva $x=x(t), t \geq 1$ deve ser a semi-reta $2 x=t+1, t \geq 1$ e a solução global desejada é

$$
u(x, t)= \begin{cases}1, & x<1+\frac{1}{2}(t-1) \\ 0, & x>1+\frac{1}{2}(t-1)\end{cases}
$$

então $u$ é descontínua ao longo da semi-reta $2 x=t+1, t \geq 1$ e as derivadas de primeira ordem de $u$ são descontínuas (alem é claro da semi-reta acima), nos segmentos de reta

$$
\begin{gathered}
x=t, \quad 0 \leq t<1 \quad e \\
x=1, \quad t=1
\end{gathered}
$$

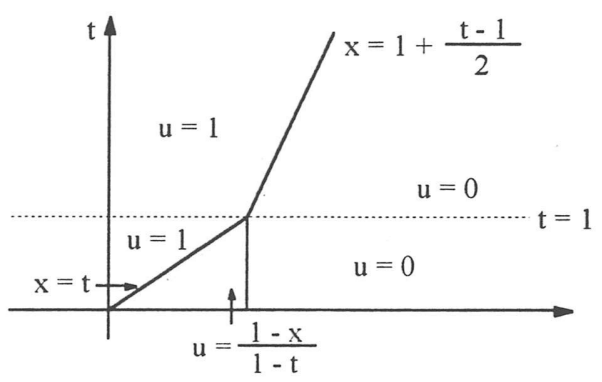

Figura 2.6

Veja Figura 2.6

Exemplo 2.2.2 Outra vez vamos considerar a equação de Burgers (2.6) com dado

$$
u_{0}(x)= \begin{cases}0, & x<0, \\ 1, & x>0 .\end{cases}
$$

$\mathrm{O}$ método das características determina a solução em todo $t>0$, exceto no setor $0<x<t$. As características são dadas como no exemplo anterior, com isto conseguimos

$$
u_{1}(x, t)= \begin{cases}0, & x<0, \quad t>0, \\ x / t, & 0<x<t, \\ 1, & x>t>0 .\end{cases}
$$


e usando o salto de descontinuidade conseguimos uma outra solução

$$
u_{2}(x, t)= \begin{cases}0, & x<t / 2 \\ 1, & x>t / 2\end{cases}
$$

Vemos que $u_{1}$ e $u_{2}$ são soluções do nosso problema. Podemos também notar que $u_{1}$ é uma função contínua. O fato de uma solução contínua poder ter valores iniciais descontínuos é uma característica de equações não lineares.

Exemplo 2.2.3 Consideremos outra vez a equação de Burger (2.2) mas agora com dado inicial

$$
u_{0}(x)= \begin{cases}-1, & x<0 \\ 1, & x>0 .\end{cases}
$$

Para cada $\alpha \geq 1$, este problema tem uma solução $u_{\alpha}$, definida por:

$$
u_{\alpha}(t, x)= \begin{cases}-1, & 2 x<(1-\alpha) t, \\ -\alpha, & (1-\alpha) t<2 x<0, \\ \alpha, & 0<2 x<(\alpha-1) t, \\ 1, & (\alpha-1) t<2 x .\end{cases}
$$


Assim os problemas (2.2), (2.10)

tem um contínuo de soluções.

Veja Figura 2.7.

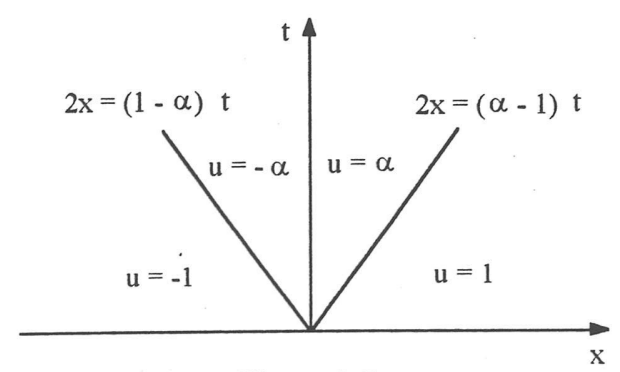

Figura 2.7

Agora, equações da forma acima (ou mais geralmente, sistemas de leis de conservação), surgem na física e então devemos ter algum mecanismo para encontrar soluções fisicamente relevantes. Assim, impomos a prióri, condições na solução que destinguem a correta uma da outra. No caso de uma equação escalar $u_{t}+f(u)_{x}=0$ $(n=1)$ onde $f^{\prime \prime}>0$, mostraremos, no próximo capítulo, que existe uma solução única que satisfaz a condição de entropia

$$
\frac{u(x+a, t)-u(x, t)}{a} \leq \frac{E}{t}, \quad a>0, \quad t>0,
$$

onde $E$ é independente de $x, t$ e $a$. Esta condição implica que se fixarmos $t>0$ e fizermos $x$ de $-\infty$ a $+\infty$ então podemos somente saltar para baixo, isto é, numa direção através da descontinuidade aqui está a razão para a entropia. Se retornarmos ao exemplo 2 vemos que (2.12) é satisfeita somente quando $\alpha=1$; isto é, $u_{1}$ é a solução ilustre. Veja (2.11).

A condição (2.12) é um bocado estranha quando encontrada pela primeira vez mas daremos um argumento, que num certo sentido, mostra que (2.12) é completamente natural.

Assim considere a equação escalar $u_{t}+f(u)_{x}=0$. Supondo que a solução é suave e tem $u_{0}^{\prime} \geq 0$

$$
u_{x}=\frac{u_{0}^{\prime}}{1+u_{0}^{\prime} f^{\prime \prime} t}
$$

Assim, se $u_{0}^{\prime}=0$ então $u_{x}=0$ e se $u_{0}^{\prime}>0$ então

$$
u_{x} \leq \frac{u_{0}^{\prime}}{u_{0}^{\prime} f^{\prime \prime} t}=\frac{1}{f^{\prime \prime} t}<\frac{E}{t}
$$

onde $E=1 / \mu$ e $\mu=\inf f^{\prime \prime} ;$ isto é $(2.3)$.

Esta perda de unicidade é encontrada em problemas relacionados com dinâmica de gas. Assim, a condição de Rankine-Hugoniot admite choques de "rarefação "; isto é, descontinuidade através do decrescimento de entropia, e naturalmente estas soluções são fisicamente inaceitáveis. 
Observação 2.2.1 A perda de unicidade ocorre em classes de funções suaves. Nos exemplos acima, a unicidade não foi perdida pelo fato de admitirmos soluções tụrbulentas do ponto de vista da regularidade o caso é um pouco mais delicado. Os bons e maus choques são indistinguiveis do ponto de vista de regularidade.

Se considerarmos outra vez (2.3) onde $f^{\prime \prime}>0$ então, como temos observado, o salto deve sempre ser em declive quando $x$ cresce; isto é, $u_{l}>u_{r}$. Se escrevermos a condição de salto na forma

$$
s=\frac{f\left(u_{l}\right)-f\left(u_{r}\right)}{u_{l}-u_{r}}=f^{\prime}(\xi)
$$

onde, $u_{l}>\xi>u_{r}$ então obtemos a desigualdade de entropia

$$
f^{\prime}\left(u_{l}\right)>s>f^{\prime}\left(u_{r}\right)
$$

este estado que é a velocidade do choque, é intermediário para a velocidade das características sobre ambos os lados do choque.

Exemplo 2.2.4 Observe que a solução suave de $u_{t}+u u_{x}=0$ também satisfaz as seguintes equações :

$$
u_{t}+\left(\frac{u^{2}}{2}\right)_{x}=0 \quad e \quad\left(\frac{u^{2}}{2}\right)_{t}+\left(\frac{u^{3}}{3}\right)_{x}=0
$$

Mas isto não é verdade para soluções descontínuas. Para ver isto, note que a primeira satisfaz a condição de salto $s\left(u_{l}-u_{r}\right)=\left(u_{l}^{2}-u_{r}^{2}\right) / 2$ ou $s=\left(u_{l}+u_{r}\right) / 2$. A segunda satisfaz a equação $s=2\left(u_{l}^{2}+u_{l} u_{r}+u_{r}^{2}\right) / 3\left(u_{l}+u_{r}\right)$. Como são obviamente diferentes, o mesmo é verdade para a classe de solução de descontinuidade.

Assim, a mesma equação diferencial pode ser escrita em duas formas divergentes diferentes, se tem o conjunto diferente de soluções fracas.

O próximo exemplo mostra que soluções fracas não são preservadas sobre transformações suaves não lineares de equações .

Exemplo 2.2.5 Consideraremos a equação singular $u_{t}+f(u)_{x}=0$ onde $f^{\prime \prime}>0 . A$ transformação não linear $u \rightarrow v=f^{\prime}(u)$ aplica soluções suaves desta equação em soluções suaves da equação de Burgers $v_{t}+v v_{x}=0$. Mas não aplica soluções descontínuas nelas mesmas. A equação original implica

$$
s=\frac{f\left(u_{l}\right)-f\left(u_{r}\right)}{u_{l}-u_{r}},
$$


enquanto a equação transformada produz

$$
s=\frac{v_{l}+v_{r}}{2}=\frac{f^{\prime}\left(u_{l}\right)+f^{\prime}\left(u_{r}\right)}{2}
$$

Como estes dois valores são geralmente diferentes vemos que se u satisfaz a equação original, $v=f^{\prime}(u)$ não é em geral a solução suave da equação de Burgers.

\subsection{Irreversibilidade}

Ilustraremos a irreversibilidade matematicamente com um simples exemplo. Para $0 \leq \varepsilon \leq 1$, definimos $u_{\varepsilon}(x, t)$ uma solução da equação

$$
u_{t}+\left(\frac{u^{2}}{2}\right)_{x}=0
$$

para $t \leq \varepsilon$, temos

$$
u_{\varepsilon}(x, t)=\left\{\begin{array}{lc}
1, & x<t-\varepsilon / 2, \\
\frac{x-\varepsilon / 2}{t-\varepsilon}, & t-\varepsilon / 2<x<\varepsilon / 2, \\
0, & x>\varepsilon / 2
\end{array}\right.
$$

enquanto para $t>\varepsilon$ definimos

$$
u_{\varepsilon}(x, t)=\left\{\begin{array}{cc}
1, & x<t / 2 \\
0, & x>t / 2
\end{array}\right.
$$

as $u_{\varepsilon}$ são descritas na figura 2.8 .
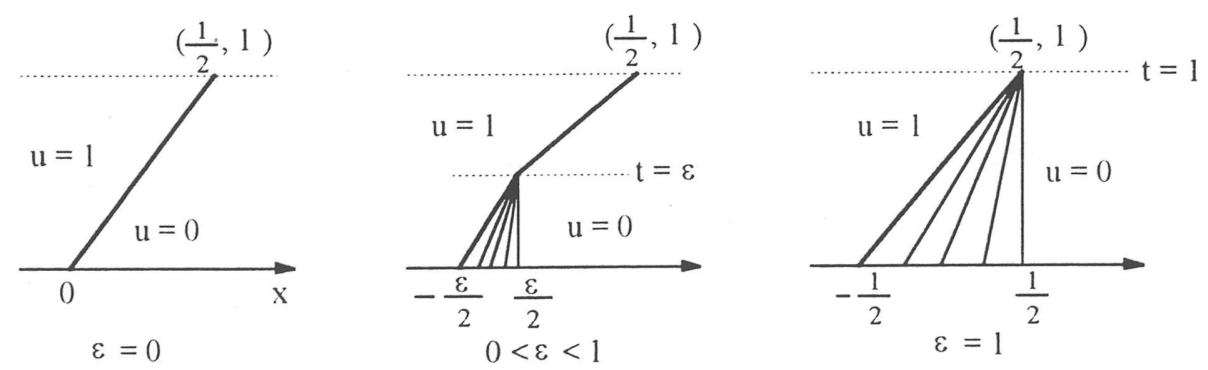

Figura 2.8

Podemos ver que as duas equações são soluções, mais ainda cada $u_{\varepsilon}$ satisfaz a 
condição de entropia (2.13). Assim elas são soluções "corretas". O ponto que queremos enfatizar aqui é que estas soluções coincidem em $t=1$

$$
u_{\varepsilon}(x, 1)= \begin{cases}1, & x<1 / 2, \\ 0, & x>1 / 2\end{cases}
$$

Em $t=1$ sabemos que um choque foi formado, não sabemos quando ele foi formado, nem podemos dizer como ele foi formado.

Enfatizamos outra vez que todas estas soluções são "certas" e pertencem a classe de soluções, provaremos no próxiṃo capítulo que são unicamente determinadas por seus valores iniciais. Mas esta unicidade é somente na direção de decrescimento de $t$. Duas soluções nesta classe que são iguais em $t=t_{0}$ devem ser iguais para todo $t>t_{0}$, mas não precisam ser iguais em $t<t_{0}$. É neste sentido que soluções de leis de conservação são irreversíveis. 


\section{Capítulo 3}

\section{Leis de conservação escalar}

\subsection{Existência de uma solução de entropia}

Consideremos a lei de conservação escalar

$$
u_{t}+f(u)_{x}=0 \quad t>0, \quad x \in \mathbb{R}
$$

com dado inicial

$$
u(x, 0)=u_{0}(x), \quad x \in \mathbb{R}
$$

Assumiremos que $u_{0} \in L_{\infty}(\mathbb{R})$ e que $f^{\prime \prime}>0$.

Definição 3.1.1 Uma solução de (3.1), (3.2) é uma função u(x,t) localmente limitada, e mensurável que satisfaz:

$$
\iint_{t>0}\left(u \phi_{t}+f(u) \phi_{x}\right) d x d t+\int_{t=0} u_{0} \phi d x=0
$$

para toda função teste $\phi \in C_{0}^{1}$.

O objetivo desta secção é provar o seguinte teorema:

Teorema 3.1.1 Sejam $u_{0} \in L_{\infty}(\mathbb{R})$ e $f \in C^{2}(\mathbb{R})$ com $f^{\prime \prime}>0$ sobre $\left\{u:|u| \leq\left\|u_{0}\right\|_{\infty}\right\}$. Então existe uma solução u de (3.1) e (3.2) com as seguintes propriedades: 
(a) $|u(x, t)| \leq\left\|u_{0}\right\|_{\infty} \equiv M,(x, t) \in \mathbb{R} \times \mathbb{R}_{+}$

(b) Existe uma constante $E>0$, que depende de $M, \mu=\min \left\{f^{\prime \prime}(u):|u| \leq\left\|u_{0}\right\|_{\infty}\right\}$ e $A=\max \left\{f^{\prime \prime}(u):|u| \leq\left\|u_{0}\right\|_{\infty}\right\}$ tal que para todo $a>0, t>0$ e $x \in \mathbb{R}$

$$
\frac{u(x+a, t)-u(x, t)}{a}<\frac{E}{t}
$$

(c) u é estável e depende continuamente de $u_{0}$ no seguinte sentido:

Se $v_{0} \in L_{\infty}(\mathbb{R})$ com $\left\|v_{0}\right\|_{\infty} \leq\left\|u_{0}\right\|_{\infty}$ e v é a correspondente solução de (3.1) com dado inicial $v_{0}$, então para todo $x_{1}$ e $x_{2} \in \mathbb{R}$, com $x_{1}<x_{2}$ e todo $t>0$

$$
\int_{x_{1}}^{x_{2}}|u(x, t)-v(x, t)| d x \leq \int_{x_{1}-A t}^{x_{2}+A t}\left|u_{0}(x)-v_{0}(x)\right| d x
$$

Antes de provarmos o teorema, faremos algumas observações :

Observação 3.1.1 Podemos notar que (3.5) mostra que a solução é estável e única. A unicidade é obtida da condição de entropia (3.4), como mostraremos na próxima secção .

Observação 3.1.2 A condição de entropia (3.4) pode ser olhada como um resultado de regularidade, já que para todo $t>0$, a solução $u(., t)$ é de variação total localmente limitada. Para ver isto, seja $c_{1}$ uma constante tal que $c_{1}>\frac{E}{t}$ e seja $v(x, t)=u(x, t)-c_{1}$. Então se $a>0,(3.4)$ implica que

$$
v(x+a, t)-v(x, t)=u(x+a, t)-u(x, t)-c_{1} a \leq a\left(\frac{E}{t}-c_{1}\right)<0
$$

$\operatorname{logo} v$ é uma função extritamente decrescente e portanto tem variação total localmente limitada. () mesmo acontece para a função $c_{1}$ assim nossa é válida.

Assim, embora o dado inicial esteja em $L_{\infty}$ para $t=0$, a solução torna-se imediatamente regular em $t>0$. Então podemos concluir que $u(., t)$ tem um número enumerável de saltos de descontinuidades, que é diferenciável quase sempre, etc... .Assim, a propriedade de regularização da solução é de fato um fenômeno puramente não linear. Por outro lado, se admitirmos que soluções podem ser construídas via o método de viscosidade então a solução é favoravelmente regular, já que sabemos que soluções de equações parabólicas exibem um alto grau de regularidade.

Vamos definir agora o método da diferença que será importante na obtenção da solução 
Consideremos o semiplano $t \geq 0$ recoberto por uma grade $t=k h, x=n l, n=$ $0, \pm 1, \pm 2, \cdots . k=0,1,2, \cdots$. onde $h=\Delta t>0, l=\Delta x>0$.

Consideremos a seguinte aproximação para (3.1):

$$
\frac{u_{n}^{k+1}-\left(u_{n+1}^{k}+u_{n-1}^{k}\right) / 2}{h}+\frac{f\left(u_{n+1}^{k}\right)-f\left(u_{n-1}^{k}\right)}{2 l}=0
$$

definida sobre nossa grade, onde estamos usando a notação

$$
u_{\beta}^{\alpha}=u(\beta l, \alpha h)
$$

e

$$
u_{n}^{0}=u_{0}(n l) \quad n=0, \pm 1, \pm 2, \cdots
$$

(poderíamos ter considerado

$$
\left.u_{n}^{0}=\left(\frac{1}{l}\right) \int_{\left(n-\frac{1}{2}\right) l}^{\left(n+\frac{1}{2}\right) l} u_{0}(x) d x\right)
$$

Sejam

$$
\begin{gathered}
M=\left\|u_{0}\right\|_{\infty} \\
A=\max _{|u| \leq M}\left|f^{\prime}(u)\right|
\end{gathered}
$$

$\mathrm{e}$

$$
\mu=\min _{|u| \leq M} f^{\prime \prime}(u)
$$

observemos que $A>0$ e $\mu>0$.

Éscolhemos os parâmetros da malha $h$ e $l$ tal que a seguinte condição ocorra

$$
\frac{A h}{l} \leq 1
$$

Lema 3.1.1 Para todo $n \in Z$ e $k \in Z_{+}$temos $\left|u_{n}^{k}\right| \leq M$. 
Prova: Escrevemos a equação diferença (3.1) na forma:

$$
\begin{aligned}
u_{n}^{k+1} & =\frac{-h}{2 l}\left(f\left(u_{n+1}^{k}\right)-f\left(\begin{array}{l}
k \\
n-1
\end{array}\right)\right)+\frac{1}{2}\left(u_{n+1}^{k}+u_{n-1}^{k}\right) \\
& =\frac{-h}{2 l} f^{\prime}\left(\theta_{n}^{k}\right)\left(u_{n+1}^{k}-u_{n-1}^{k}\right)+\frac{1}{2}\left(u_{n+1}^{k}+u_{n-1}^{k}\right)
\end{aligned}
$$

onde $u_{n-1}^{k} \leq \theta_{n}^{k} \leq u_{n+1}^{k}$. Então

$$
u_{n}^{k+1}=\left(\frac{1}{2}+\frac{h}{2 l} f^{\prime}\left(\theta_{n}^{k}\right)\right) u_{n-1}^{k}+\left(\frac{1}{2}-\frac{h}{2 l} f^{\prime}\left(\theta_{n}^{k}\right)\right) u_{n+1}^{k}
$$

Por indução sobre $\left|u_{n}^{k}\right| \leq M$ para todo $n \in \mathbf{Z}$ e $k \in \mathbf{Z}_{+}$vemos que (3.9) implica

$$
\frac{1}{2} \pm \frac{h}{2 l} f^{\prime}\left(\theta_{n}^{k}\right) \geq 0
$$

Assim,

$$
\left|u_{n}^{k+1}\right| \leq M\left(\frac{1}{2}+\frac{h}{2 l} f^{\prime}\left(\theta_{n}^{k}\right)\right)+M\left(\frac{1}{2}-\frac{h}{2 l} f^{\prime}\left(\theta_{n}^{k}\right)\right)=M .
$$

Lema 3.1.2 Se J é uma constante e w é a solução da E.D.O. $\frac{d w}{d t}=-c w^{2}$ com dado inicial $w(0)=w_{0}$ então

$$
w(t)=\frac{1}{c t+1 / M^{0}}
$$

e se

$$
\frac{J-w(n h)}{h} \leq w^{\prime}(n h)
$$

então $J \leq w(n h+h)$

\section{Prova:}


Note que $w(t)>0$ se $t \geq 0 \operatorname{assim} w^{\prime}(t)=$ $-c w^{2}<0$ e $w^{\prime \prime}(t)=-c 2 w w^{\prime}>0$ em $t \geq 0$.

Seja $\alpha$ e $\beta$ os ângulos indicados na Figura 3.1. Como $\alpha \geq \beta$, temos

$$
\frac{w(n h+h)-w(n h)}{h} \geq w^{\prime}(n h) \geq \frac{J-w(n h)}{h}
$$

Logo $J \leq w(n h+h)$.

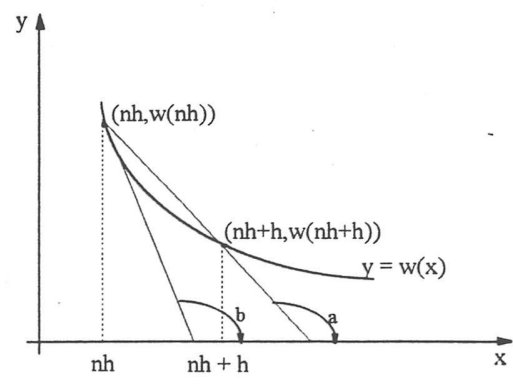

Figura 3.1

O lema a seguir é uma versão discreta da condição de entropia (3.4)

Lema 3.1.3 (Condição de entropia) Se $c=\min (\mu / 2, A / 4 M)$ então para $n \in Z, k \in$ $Z_{+}$,

$$
\frac{u_{n}-u_{n-2}^{k}}{2 l} \leq \frac{E}{k h} \quad \text { onde } \quad E=\frac{1}{c}
$$

Prova: Seja

$$
Z_{n}^{k}=\frac{u_{n}^{k}-u_{n-2}^{k}}{2 l}
$$

Então da equação diferença (3.6) temos:

$$
\begin{aligned}
Z_{n}^{k+1} & =\frac{1}{2}\left(Z_{n+1}^{K}+Z_{n-1}^{k}\right)+2 f\left(u_{n-1}^{k}\right) \frac{h}{4 l^{2}}-\frac{\left[f\left(u_{n+1}^{k}\right)-f\left(u_{n-3}^{k}\right)\right]}{4 l^{2}} h \\
& =\frac{1}{2}\left(Z_{n+1}^{K}+Z_{n-1}^{k}\right)-\frac{h}{4 l^{2}}\left[f\left(u_{n-3}^{k}\right)-f\left(u_{n-1}^{k}\right)\right]-\frac{h}{4 l^{2}}\left[f\left(u_{n+1}^{k}\right)-f\left(u_{n-1}^{k}\right)\right] \\
& =\frac{1}{2}\left(Z_{n+1}^{K}+Z_{n-1}^{k}\right)-\frac{h}{4 l^{2}}\left[f ^ { \prime } \left(u_{n-1}^{k}\left(u_{n-3}^{k}-u_{n-1}^{k}\right)\right.\right. \\
& \left.+\frac{1}{2} f^{\prime \prime}\left(\theta_{1}\right)\left(u_{n-3}^{k}-u_{n-1}^{k}\right)^{2}\right]-\frac{h}{4 l^{2}}\left[f^{\prime}\left(u_{n-1}^{k}\left(u_{n+1}^{k}-u_{n-1}^{k}\right)+\frac{1}{2} f^{\prime \prime}\left(\theta_{2}\right)\left(u_{n+1}^{k}-u_{n-1}^{k}\right)^{2}\right]\right.
\end{aligned}
$$

onde $u_{n-3}^{k} \leq \theta_{1} \leq u_{n-1}^{k}$, e $u_{n-1}^{k} \leq \theta_{2} \leq u_{n+1}^{k}$.

Segue-se

$$
Z_{n}^{k+1}=\left(\frac{1}{2}+\frac{h}{2 l} f^{\prime}\left(u_{n-1}^{k}\right)\right) Z_{n-1}^{k}+\left(\frac{1}{2}-\frac{h}{2 l} f^{\prime}\left(u_{n-1}^{k}\right)\right) Z_{n+1}^{k}-\frac{h}{2}\left[\left(Z_{n-1}^{k}\right)^{2} f^{\prime \prime}\left(\theta_{1}\right)+\left(Z_{n+1}^{k}\right)^{2} f^{\prime \prime}\left(\theta_{2}\right)\right]
$$

Definamos: 


$$
\widetilde{Z}_{n}^{k}=\max \left\{Z_{n-1}^{k}, Z_{n+1}^{k}, 0\right\}
$$

então se $\widetilde{Z}_{n}^{k}=0$ a estimativa (3.11) certamente acontece pois

$$
Z_{n}^{k} \leq \widetilde{Z}_{n}^{k}=0 \leq E / k h
$$

Podemos, portanto assumir que $\widetilde{Z}_{n}^{k} \neq 0$.

Suponhamos que $\widetilde{Z}_{n}^{k}=Z_{n+1}^{k}$. Usando (3.9) temos

$$
\begin{aligned}
Z_{n}^{k+1} & =\left(\frac{1}{2}+\frac{h}{2 l} f^{\prime}\left(u_{n-1}^{k}\right)\right) Z_{n-1}^{k}+\left(\frac{1}{2}-\frac{h}{2 l} f^{\prime}\left(u_{n-1}^{k}\right)\right) Z_{n+1}^{k}-\frac{h}{2}\left[\left(Z_{n-1}^{k}\right)^{2} f^{\prime \prime}\left(\theta_{1}\right)+\left(Z_{n+1}^{k}\right)^{2} f^{\prime \prime}\left(\theta_{2}\right)\right. \\
& \leq\left(\frac{1}{2}+\frac{h}{2 l} f^{\prime}\left(u_{n-1}^{k}\right)\right) Z_{n-1}^{k}+\left(\frac{1}{2}-\frac{h}{2 l} f^{\prime}\left(u_{n-1}^{k}\right)\right) Z_{n+1}^{k}-\frac{h \mu}{2}\left(\left(Z_{n-1}^{k}\right)^{2}+\left(Z_{n+1}^{k}\right)^{2}\right) \\
& \leq\left(\frac{1}{2}+\frac{h}{2 l} f^{\prime}\left(u_{n-1}^{k}\right)\right) Z_{n-1}^{k}+\left(\frac{1}{2}-\frac{h}{2 l} f^{\prime}\left(u_{n-1}^{k}\right)\right) Z_{n+1}^{k}-\operatorname{ch}\left(\left(Z_{n-1}^{k}\right)^{2}+\left(Z_{n+1}^{k}\right)^{2}\right) \\
& \leq\left(\frac{1}{2}+\frac{h}{2 l} f^{\prime}\left(u_{n-1}^{k}\right)\right) \widetilde{Z}_{n}^{k}+\left(\frac{1}{2}-\frac{h}{2 l} f^{\prime}\left(u_{n-1}^{k}\right)\right) \widetilde{Z}_{n}^{k}-\operatorname{ch}\left(Z_{n+1}^{k}\right)^{2},
\end{aligned}
$$

$\log \mathrm{O}$

$$
Z_{n}^{k+1} \leq \widetilde{Z}_{n}^{k}-\operatorname{ch}\left(Z_{n}^{k}\right)^{2}+\frac{h}{2 l}\left[f^{\prime}\left(u_{n-1}^{k}\right)-f^{\prime}\left(u_{n+1}^{k}\right] \widetilde{Z}_{n}^{k} \leq \widetilde{Z}_{n}^{k}-\operatorname{ch}\left(Z_{n}^{k}\right)^{2}\right.
$$

obtendo-se

$$
Z_{n}^{k+1} \leq \widetilde{Z}_{n}^{k}-\operatorname{ch}\left(Z_{n}^{k}\right)^{2}
$$

Sabemos pelo lema (3.1.1) que $\left|u_{n}^{k}\right| \leq M$. Usando (3.9) e o fato que $c \leq A / 4 M$ temos

$$
Z_{n}^{k}=\left|Z_{n}^{k}\right| \leq \frac{\left|u_{n}^{k}\right|+\left|u_{n-2}^{k}\right|}{2 l} \leq \frac{M}{l} \leq \frac{M}{A h} \leq\left(\frac{M}{h}\right)\left(\frac{1}{4 M c}\right)=\frac{1}{4 c h}
$$

ou ainda

$$
Z_{n}^{k} \leq \frac{1}{4 c h}
$$


Seja

$$
M^{k}=\max _{n}\left\{\widetilde{Z}_{n}^{k}\right\}
$$

Note que $M^{k}>0$. Considere $\phi(y)=y-c h y^{2}$. Como $\phi^{\prime}=1-2 c h y, \phi$ é uma função crescente se $y<\frac{1}{2 c h}$. Mas de (3.13) temos

$$
\widetilde{Z}_{n}^{k} \leq M^{k} \leq \frac{1}{4 c h}<\frac{1}{2 c h}
$$

$\operatorname{assim} \phi\left(\widetilde{Z}_{n}^{k}\right) \leq \phi\left(M^{k}\right)$ e com isto segue-se

$$
\widetilde{Z}_{n}^{k}-\operatorname{ch}\left(\widetilde{Z}_{n}^{k}\right)^{2} \leq M^{k}-\operatorname{ch}\left(M^{k}\right)^{2}
$$

logo de (3.12) concluímos que $Z_{n}^{k+1} \leq M^{k}-\operatorname{ch}\left(M^{k}\right)^{2}$ para todo $n \in \mathbf{Z}$ assim

$$
M^{k+1} \leq M^{k}-\operatorname{ch}\left(M^{k}\right)^{2}
$$

Agora consideremos a E.D.O. definida no lema anterior com $w_{0}=M^{0}$. Se mostrarmos que para todo $k$,

$$
M^{k} \leq w(k h)
$$

então

$$
Z_{n}^{k} \leq M^{k} \leq w(k h)=\frac{1}{c k h+1 / M^{0}} \leq \frac{1}{c k h}=\frac{E}{k h}
$$

e isto é (3.11), logo resta-nos provar (3.16) por indução, se $k=0$, então $M^{0} \leq w(0)$.

Suponhamos que (3.16) acontece para $k=n$, mostremos que vale para $k=n+1$. De (3.15) temos

$$
M^{n+1} \leq M^{n}-\operatorname{ch}\left(M^{n}\right)^{2}
$$

mas também

$$
M^{n} \leq w(n h)=\frac{1}{c n h+1 / M^{0}} \leq \frac{1}{c n h+c h} \leq \frac{1}{2 c h}
$$


já que (3.16) ocorre e $n \geq 1$.

Como $\phi$ é monótona em $(0,1 / c h), \phi\left(M^{n}\right) \leq \phi(w(n h))$ temos

$$
M^{n+1} \leq M^{n}-\operatorname{ch}\left(M^{n}\right)^{2} \leq w(n h)-\operatorname{chw}(n h)^{2}
$$

Assim

$$
\frac{M^{n+1}-w(n h)}{h} \leq-c w(n h)^{2}=w^{\prime}(n h)
$$

Mas do Lema 3.1.2 $M^{n+1} \leq w(n h+h)$ então

$$
Z_{n}^{k} \leq \frac{E}{k h}
$$

Lema 3.1.4 (Estimativa de espaço) Para qualquer $R>0$ e kh $\geq \alpha>0$, existe uma constante $c$ dependendo de $R$ e $\alpha$, mas independente de $h$ el, tal que:

$$
\sum_{|n| \leq R / l}\left|u_{n+2}^{k}-u_{n}^{k}\right| \leq c
$$

(Aqui, e no que se segue, a somatória é sobre todo $n \in \mathbf{Z}$, para o qual $|n| \leq R / l$.)

Prova: Seja $v_{n}^{k}=u_{n}^{k}-c_{1} n l$ onde $c_{1}$ é escolhido tal que $E / \alpha<c_{1}$. Então usando (3.11) segue-se

$$
\begin{aligned}
v_{n+2}^{k}-v_{n}^{k} & =u_{n+2}^{k}-u_{n}^{k}-2 c_{1} l \\
& \leq \frac{2 l E}{k h}-2 c_{1} l \\
& \leq 2 l\left(\frac{E}{\alpha}-c_{1}\right)<0 .
\end{aligned}
$$


Assim,

$$
\begin{aligned}
\sum_{|n| \leq R / l}\left|u_{n+2}^{k}-u_{n}^{k}\right| & =\left|u_{2}^{k}-u_{0}^{k}\right|+\sum_{0<|n|<R / l}\left|v_{n+2}^{k}-v_{n}^{k}+2 c_{1} l\right| \\
& \leq 2 M+\sum_{0<|n|<R / l}\left|v_{n+2}^{k}-v_{n}^{k}\right|+\sum_{0<|n|<R / l} 2 c_{1} l \\
& \leq 2 M+\sum_{0<|n|<R / l}\left|v_{n+2}^{k}-v_{n}^{k}\right|+2 c_{1} l\left(\frac{2 R}{l}\right) \\
& \leq 2 M+4 \max _{|n| \leq R / l}\left|v_{n}^{k}\right|+4 c_{1} R \\
& \leq 2 M+4 M+2 c_{1} R+4 c_{1} R \\
& =6 M+6 c_{1} R=c
\end{aligned}
$$

Observação 3.1.3 O próximo lema mostra que as aproximações $\left\{u_{n}^{k}\right\}$ são $L_{1} \operatorname{Lipschitz}$ (Localmente) em $k$.

Lema 3.1.5 (Estimativa de tempo) Se $h / l \geq \delta>0$, e $h, l \leq 1$, então existe $L>0$, independente de $h$ e l tal que se $k>p$, onde $k-p$ é par e ph $\geq \alpha>0$ então

$$
\sum_{|n| \leq R / l}\left|u_{n}^{k}-u_{n}^{p}\right| l \leq L(k-p) h
$$

Prova: Expressaremos $u_{n}^{k}$ em termos de $u_{n}^{p}, k-p$ par. Para fazermos isto consideraremos a equação diferença (3.6) na forma (3.11)

$$
\begin{aligned}
u_{n}^{k} & =-\frac{h}{2 l} f^{\prime}\left(\theta_{n}^{k-1}\left(u_{n+1}^{k-1}-u_{n-1}^{k-1}\right)+\frac{1}{2}\left(u_{n+1}^{k-1}+u_{n-1}^{k-1}\right)\right. \\
& =\left(\frac{1}{2}+f^{\prime}\left(\theta_{n}^{k+1}\right) \frac{h}{2 l}\right) u_{n-1}^{k-1}+\left(\frac{1}{2}+f^{\prime}\left(\theta_{n}^{k-1}\right) \frac{h}{2 l}\right) u_{n+1}^{k-1},
\end{aligned}
$$




$$
u_{n}^{k}=a_{n, n-1}^{k, k-1} u_{n-1}^{k-1}+a_{n, n+1}^{k, k-1} u_{n+1}^{k-1}
$$

onde, $a_{n, n-1}^{k, k-1}+a_{n, n+1}^{k, k-1}=1$, e $a_{n, n-1}^{k, k-1}, a_{n, n+1}^{k, k-1}>0$.

Aplicando estes valores para $u_{n-1}^{k-1}$ e $u_{n+1}^{k-1}$ temos a fórmula:

$$
u_{n}^{k+1}=A u_{n-2}^{k-1}+B u_{n}^{k-1}+C u_{n+2}^{k-1},
$$

onde $A+B+C=1$ e $A, B, C \geq 0$. Daí

$$
\left|u_{n}^{k+1}-u_{n}^{k-1}\right| \leq A\left|u_{n-2}^{k-1}-u_{n}^{k-1}\right|+C\left|u_{n+2}^{k-1}-u_{n}^{k-1}\right|
$$

Se multiplicarmos esta equação por $\Delta x$ e somarmos conseguiremos

$$
\sum_{|n| \leq R / l}\left|u_{n}^{k+1}-u_{n}^{k-1}\right| \Delta x \leq 2 c \Delta x
$$

onde $c$ é a constante em (3.17). Agora se $k-p$ é par a desigualdade triangular implica

$$
\begin{aligned}
\sum_{|n| \leq R / l}\left|u_{n}^{k}-u_{n}^{p}\right| \Delta x & \leq \sum_{|n| \leq R / l}\left(\left|u_{n}^{k}-u_{n}^{k-2}\right|+\left|u_{n}^{k-2}-u_{n}^{k-4}\right|+\ldots+\left|u_{n}^{k-2(n-1)}-u_{n}^{p}\right|\right) \Delta x \\
& \leq \frac{(k-p)}{2} 2 c \Delta x=(k-p) \Delta x \\
& \leq \frac{c}{\delta}(k-p) \Delta t
\end{aligned}
$$

fazendo $L=c / \delta$ provamos o lema.

Observação 3.1.4 O autor afirma que o lema é verdade para o caso $k-p$ ímpar. 
Lema 3.1.6 (Estabilidade) Sejam $\left\{u_{n}^{k}\right\}$ e $\left\{v_{n}^{k}\right\}$ soluções da equação diferença finita(3.16) correspondentes aos valores iniciais $\left\{u_{0}\right\}$ e $\left\{v_{0}\right\}$, respectivamente, com $\sup _{n}\left|u_{n}^{0}\right| \leq M$ $e \sup _{n}\left|v_{n}^{0}\right| \leq M$. Então se $k>0$ temos

$$
\sum_{|n| \leq N}\left|u_{n}^{k}-v_{n}^{k}\right| l \leq \sum_{|n| \leq N+k}\left|u_{0}-v_{0}\right| l
$$

Prova: Seja $w_{n}^{k}=u_{n}^{k+1}-v_{n}^{k+1}$. Então de (3.16) temos

$$
\begin{aligned}
w_{n}^{k} & =u_{n}^{k+1}-v_{n}^{k+1}=\frac{u_{n+1}^{k}+u_{n-1}^{k}}{2}-\frac{h}{2 l}\left[f\left(u_{n+1}^{k}\right)-f\left(u_{n-1}^{k}\right)\right] \\
& -\frac{v_{n+1}^{k}+v_{n-1}^{k}}{2}+\frac{h}{2 l}\left[f\left(v_{n+1}^{k}\right)-f\left(v_{n-1}^{k}\right)\right] \\
& =\frac{u_{n+1}^{k}-v_{n+1}^{k}}{2}-\frac{h}{2 l}\left[f\left(u_{n+1}^{k}\right)-f\left(v_{n+1}^{k}\right)\right] \\
& +\frac{u_{n-1}^{k}-v_{n-1}^{k}}{2}+\frac{h}{2 l}\left[f\left(u_{n-1}^{k}\right)-f\left(v_{n-1}^{k}\right)\right] \\
& =\frac{1}{2} w_{n+1}^{k}-\frac{h}{2 l} w_{n+1}^{k} f^{\prime}\left(\theta_{n+1}^{k}\right)+\frac{1}{2} w_{n-1}^{k} \\
& +\frac{h}{2 l} w_{n-1}^{k} f^{\prime}\left(\theta_{n-1}^{k}\right),
\end{aligned}
$$

onde $u_{n+1}^{k} \leq \theta_{n+1}^{k} \leq v_{n=1}^{k}$. Assim

$$
w_{n}^{k+1}=\left[\frac{1}{2}-\frac{h}{2 l} f^{\prime}\left(\theta_{n+1}^{k}\right)\right] w_{n+1}^{k}+\left[\frac{1}{2}+\frac{h}{2 l} f^{\prime}\left(\theta_{n-1}^{k}\right)\right] w_{n-1}^{k} .
$$

De (3.20), temos que os coeficientes de $w_{n+1}^{k}$ e $w_{n-1}^{k}$ são ambos não negativos assim 


$$
\begin{aligned}
\sum_{|n| \leq N}\left|w_{n}^{k+1}\right| & \leq \sum_{|n| \leq N}\left[\frac{1}{2}-\frac{h}{2 l} f^{\prime}\left(\theta_{n+1}^{k}\right)\right]\left|w_{n+1}^{k}\right| \\
& \leq \sum_{|n| \leq N}\left[\frac{1}{2}+\frac{h}{2 l} f^{\prime}\left(\theta_{n-1}^{k}\right)\right]\left|w_{n-1}^{k}\right| \\
& =\sum_{m=1-N}^{1+N}\left[\frac{1}{2}-\frac{h}{2 l} f^{\prime}\left(\theta_{m}^{k}\right)\right]\left|w_{m}^{k}\right|+\sum_{m=-1-N}^{N-1}\left[\frac{1}{2}+\frac{h}{2 l} f^{\prime}\left(\theta_{m}^{k}\right)\right]\left|w_{m}^{k}\right| \\
& \leq \sum_{|m| \leq N+1}\left[\frac{1}{2}-\frac{h}{2 l} f^{\prime}\left(\theta_{m}^{k}\right)\right]\left|w_{m}^{k}\right|+\sum_{|m| \leq N+1}\left[\frac{1}{2}+\frac{h}{2 l} f^{\prime}\left(\theta_{m}^{k}\right)\right]\left|w_{m}^{k}\right| \\
& =\sum_{|m| \leq N+1}\left|w_{m}^{k}\right|
\end{aligned}
$$

que inplica (3.20)

Vamos agora construir uma subfamília de funções $\left\{U_{h, l}\right\}$ de $\left\{U_{n}^{k}\right\}$ da seguinte forma

$$
U_{h, l}(x, t)=u_{n}^{2 k}, \quad \text { se } \quad n l \leq x<(n+1) l, \quad k h \leq t<(k+1) h .
$$

Assim o valor de $U_{h, l}$ no retângulo $n l \leq x<(n+1) l, k h \leq t<(k+1) h$, é o valor da aproximação no ponto $(n l, k h)$. Veja Figura 3.2.

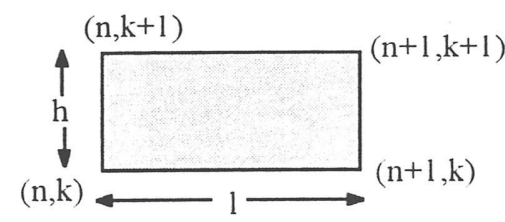

Figura 3.2

Mostraremos que o conjunto de funções $\left\{U_{h, l}\right\}$ é compacto na topologia de $L_{1}$.

Lema 3.1.7 Existe uma sequência $\left\{U_{h_{i}, l_{i}}\right\} \subset\left\{U_{h, l}\right\}$ que converge para uma função mensurável $u(x, t)$ no sentido que para todo $R>0, t>0$ e $T>0$,

$$
\begin{gathered}
\int_{|x| \leq R}\left|U_{h_{i}, l_{i}}-U(x, t)\right| d x \rightarrow 0 \\
\int_{0 \leq t \leq T} \int_{|x| \leq R}\left|U_{h_{i}, l_{i}}-U(x, t)\right| d x d t \rightarrow 0
\end{gathered}
$$

com $i \rightarrow \infty$ (isto é, $\left.\left(h_{i}, l_{i}\right) \rightarrow(0,0)\right)$. Mais ainda a função limite satisfaz

$$
\sup _{x \in \mathbf{R} t>0}|u(x, t)| \leq M
$$

e a desigualdade de estabilidade (3.5). 
Prova: Do lema (3.1.1) temos $\left|u_{n}^{2 k}\right| \leq M$. Mas $u_{n}^{2 k}(x, t)=U_{h, l}(x, t)$. Logo, $\left|U_{h, l}\right| \leq M$, portanto o conjunto de funções $\left\{U_{h, l}\right\}$ consideradas como funções de $x$ são unifơrmemente limitadas em cada intervalo limitado sobre qualquer reta $t=$ const $>0$.

Além disso

$$
\begin{aligned}
V\left(\left\{U_{h, l}\right\}\right) & =\sup \left\{\sum_{j=0}^{n-1}\left|U_{h, l}\left(x_{j+1}, t\right)-U_{h, l}\left(x_{j}, t\right)\right|\right\} \\
& =\sup \left\{\sum_{j=0}^{n-1}\left|u_{n}^{2 k}\left(x_{j+1}, t\right)-u_{n}^{2 k}\left(x_{j}, t\right)\right|\right\} \\
& \leq \sup \left\{\sum_{|n| \leq R / l}\left|u_{n+2}^{k}-u_{n}^{k}\right|\right\} \leq c
\end{aligned}
$$

pelo Lema(3.5). Logo, $\left\{U_{h, l}\right\}$ tem variação total uniformemente limitada em cada intervalo limitado sobre qualquer reta $t=$ const $>0$. (a uniformidade sendo de fato, com respeito a $h$ e $l$ ).

Pelo teorema (1.2.1) podemos encontrar uma subsequência $\left\{U_{h, l}^{\prime}\right\}$ que converge em cada ponto sobre qualquer intervalo limitado desta reta e por um processo de diagonalização podemos construir uma subsequência $\left\{U^{\prime \prime}{ }_{h, l}\right\}$ de $\left\{U_{h, l}^{\prime}\right\}$ que converge em todo ponto desta reta $t=$ const $>0, \operatorname{com} h$ e $l$ tendendo a zero.

Seja $\left\{t_{m}\right\}$ um subconjunto enumerável e denso no intervalo $(0, T)$. Por um novo processo de diagonalização podemos selecionar uma subsequência $\left\{U_{h_{i}, l_{i}}\right\}$ de $\left\{U^{\prime \prime}{ }_{h, l}\right\}$ que converge em todos os pontos sobre cada reta $t=t_{m}, m=1,2, \ldots$ com $i \rightarrow \infty$, isto é, $h_{i}$ e $l_{i}$ tendendo para zero.

Seja $U_{i}=U_{h_{i}, l_{i}}$. Mostraremos que esta sequência de funções converge para cada $t \in(0, T]$ para uma função $u(x, t)$ que está definida na faixa $0<t \leq T$.

Para fazer isto, primeiro mostraremos que para todo $t \in(0, T]$,

$$
I_{i, j}(t) \equiv \int_{-x}^{x}\left|U_{i}\left(x^{\prime}, t\right)-U_{j}\left(x^{\prime}, t\right)\right| d x^{\prime} \rightarrow 0
$$

com $i, j \rightarrow \infty$, isto é, $\left\{U_{i}(., t)\right\}$ é uma sequência de Cauchy em $L_{1}(|x| \leq R)$.

Para $t \in(0, T]$ encontramos uma subsequência $\left\{t_{m_{s}}\right\} \subset\left\{t_{m}\right\}$ tal que $t_{m_{s}} \rightarrow t \mathrm{com}$ $s \rightarrow \infty$. Seja $\tau_{s}=t_{m_{s}}$. Então:

$$
\begin{aligned}
I_{i, j}(t) & \leq \int_{-x}^{x}\left|U_{i}\left(x^{\prime}, t\right)-U_{i}\left(x^{\prime}, \tau_{s}\right)\right| d x^{\prime}+\int_{-x}^{x}\left|U_{i}\left(x^{\prime}, \tau_{s}\right)-U_{j}\left(x^{\prime}, \tau_{s}\right)\right| d x^{\prime} \\
& +\int_{-x}^{x}\left|U_{j}\left(x^{\prime}, \tau_{s}\right)-U_{j}\left(x^{\prime}, t\right)\right| d x^{\prime} \equiv I_{1}+I_{2}+I_{3}
\end{aligned}
$$


Em vista da escolha de $\left\{t_{m}\right\}$ e do teorema (1.2) vemos que $I_{2} \rightarrow 0$ com $i, j \rightarrow \infty$. Agora se $[\sigma]$ denota o maior inteiro menor que $\sigma$ então pela definição de $U_{i}$ temos $U_{i}(x, t)=U_{i}\left(x,\left[t / h_{i}\right] h_{i}\right)$ assim:

$$
\begin{aligned}
I_{1} & =\int_{-x}^{x}\left|U_{i}\left(x^{\prime},\left[\frac{t}{h_{i}}\right] h_{i}\right)-U_{i}\left(x^{\prime},\left[\frac{\tau_{s}}{h_{i}}\right] h_{i}\right)\right| d x^{\prime} \\
& \leq \int_{-\left[R / l_{i}+1\right] l_{i}}^{\left[R / l_{i}+1\right] l_{i}}\left|U_{i}\left(x^{\prime},\left[\frac{t}{h_{i}}\right] h_{i}\right)-U_{i}\left(x^{\prime},\left[\frac{\tau_{s}}{h_{i}}\right] h_{i}\right)\right| d x^{\prime} \\
& \leq \sum_{|n| \leq R / l_{i}+1} \int_{n l_{i}}^{(n+1) l_{i}}\left|U_{i}\left(x^{\prime},\left[\frac{t}{h_{i}}\right] h_{i}\right)-U_{i}\left(x^{\prime},\left[\frac{\tau_{s}}{h_{i}}\right] h_{i}\right)\right| d x^{\prime} \\
& =\sum_{|n| \leq R / l_{i}+1}\left|u_{n}^{2\left[t / h_{i}\right]}-u_{n}^{2\left[\tau_{s} / h_{i}\right]}\right| l_{i} \\
& \leq L\left|\left[\frac{t}{h_{i}}\right]-\left[\frac{\tau_{s}}{h_{i}}\right]\right| h_{i}
\end{aligned}
$$

Onde usamos o Lema(3.6).

Assim $I_{1} \leq L\left|\left[\frac{t}{h_{i}}\right]-\left[\frac{\tau_{s}}{h_{i}}\right]\right| h_{i}$ e de modo análogo temos $I_{3} \leq L\left|\left[\frac{t}{h_{i}}\right]-\left[\frac{\tau_{s}}{h_{i}}\right]\right| h_{i} \operatorname{logo}$

$$
I_{1}+I_{3} \leq 2 L\left|\left[\frac{t}{h_{i}}\right]-\left[\frac{\tau_{s}}{h_{i}}\right]\right| h_{i}
$$

Agora dado $\varepsilon>0$, escolhemos $\tau_{s}$ tal que $4 L\left|\left[\frac{t}{h_{i}}\right]-\left[\frac{\tau_{s}}{h_{i}}\right]\right| h_{i}<\varepsilon$. Fixado s escolhemos $i$ e $j$ tão grande que $2 I_{2}<\varepsilon$. Então para este $i$ e $j$, temos:

$$
I_{i, j}(t) \leq I_{1}+I_{2}+I_{3} \leq 2 L\left|\left[\frac{t}{h_{i}}\right]-\left[\frac{\tau_{s}}{h_{i}}\right]\right| h_{i}+I_{2} \leq \frac{\varepsilon}{2}+\frac{\varepsilon}{2}=\varepsilon
$$

Portanto $I_{i, j}(t) \leq \varepsilon$ e isto mostra (3.24).

Logo $\left\{U_{i}(x, t)\right\}$ é uma sequência de Cauchy e tem limite $u(x, t)$ para cada $t$ fixado, $0<t \leq T$. Mostraremos agora que $I_{i, j}(t) \rightarrow 0 \mathrm{com} i, j \rightarrow \infty$ uniformemente em $t$ com $0<\tau<t \leq T$.

Para ver isto dado $\varepsilon>0$ escolha um subconjunto finito $\Im \subset\left\{t_{m}\right\}$ com a propriedade que se $0 \leq t \leq T$ existe um $t_{m}$ em $\Im$ tal que $2 L\left[\frac{t}{h_{i}}\right]-\left[\frac{t_{m}}{h_{i}}\right] \mid h_{i} \leq \frac{\varepsilon}{2}$. Se escolhermos $i, j$ tão grande que $2 L_{2}<\varepsilon$ para todo $t_{m} \in \Im$ então para estes $i, j$ temos $I_{i, j}(t)<\varepsilon$. Isto 
dá a uniformidade desejada em $t$.

Usando a convergência uniforme temos que para qualquer $\tau, 0<\tau \leq T$,

$$
\int_{\tau}^{T} I_{i, j}(t) d t \rightarrow 0
$$

$\operatorname{com} i, j \rightarrow \infty$. Seja

$$
\begin{aligned}
\int_{0}^{T} \int_{-R}^{R}\left|U_{i}(x, t)-U_{j}(x, t)\right| d x d t & =\int_{0}^{\tau} \int_{-R}^{R}\left|U_{i}(x, t)-U_{j}(x, t)\right| d x d t \\
& +\int_{\tau}^{T} \int_{-R}^{R}\left|U_{i}(x, t)-U_{j}(x, t)\right| d x d t
\end{aligned}
$$

Se escolhermos $\tau, 0<\tau \leq T$ tão pequeno que $8 M R \tau<\varepsilon$ então para este $\tau$ fixado, tomamos $i, j$ tão grande tal que

$$
\int_{\tau}^{T} \int_{-x}^{x}\left|U_{i}\left(x^{\prime}, t\right)-U_{j}\left(x^{\prime}, t\right)\right| d x^{\prime} d t<\varepsilon
$$

Isto segue de (3.27). Então para estes $i, j$, temos

$$
\int_{0}^{T} I_{i, j}(t)<\varepsilon
$$

Portanto $\left|U_{i}(x, t)-U_{j}(x, t)\right|$ é uma sequência de Cauchy em $[-R, R] \times[0, T]$. Segue que $u(x, t)$ é mensurável e que (3.23) acontece.

Mais ainda, como a convergência local em $L_{1}$ implica convergência pontual quase sempre de uma subsequência, vemos que como cada $\left|U_{i}\right| \leq M$ a função limite sa-tisfaz a mesma desigualdade onde, se necessário, redefinimos $u$ num conjunto de medida zero.

Finalmente (3.5) acontece em vista do Lema (3.1.6) pois $\exists N \in \mathbf{N} \operatorname{com}\left[x_{1}, x_{2}\right] \subset$ $[-N l,(-N+1) l] \subset[-N l, N l]$ assim 


$$
\begin{aligned}
\int_{x_{1}}^{x_{2}}|u(x, t)-v(x, t)| d x & \leq \int_{-N l}^{N l}|u(x, t)-v(x, t)| d x=\sum_{|n| \leq N} \int_{n l}^{(n+1) l}|u(x, t)-v(x, t)| d x \\
& =\sum_{|n| \leq N} \int_{n l}^{(n+1) l}\left|u_{n}^{2 k}-v_{n}^{2 k}\right| \leq l\left|u_{n}^{2 k}-v_{n}^{2 k}\right| \\
& \leq \sum_{|n| \leq N}\left|u_{n}^{2 k}-v_{n}^{2 k}\right| l \leq \sum_{|n| \leq N+k}\left|u_{n}^{0}-v_{n}^{0}\right| l \\
& \leq \sum_{|n| \leq N} \int_{n l}^{(n+1) l}\left|u_{0}(x)-v_{0}(x)\right| d x=\int_{x_{1}}^{x_{2}}\left|u_{0}(x)-v_{0}(x)\right| d x \\
& \leq \int_{-N l}^{N l}\left|u_{0}(x)-v_{0}(x)\right| d x \leq \int_{x_{1}-A t}^{x_{2}+A t}\left|u_{0}(x)-v_{0}(x)\right| d x
\end{aligned}
$$

$\operatorname{com} u_{n}^{0}=\frac{1}{l} \int_{n l}^{(n+1) l} u_{0}(x) d x$

Lema 3.1.8 A função u $(x, t)$ no lema anterior satifaz a desigualdade de entropia.

Prova: É suficiente mostrar que se $\left(x_{1}-x_{2}\right)>2 l_{i}$ e $t>h_{i}$,

$$
\frac{U_{i}\left(x_{1}, t\right)-U_{i}\left(x_{2}, t\right)}{x_{1}-x_{2}}<\frac{2 E^{\prime}}{t-h_{i}}
$$

onde os $U_{i}^{\prime} s$ são definidos como na prova do Lema anterior e a constante $E^{\prime}=E / 2$ onde $E$ é definido no Lema (3.1.3). Se (3.29) acontece, conseguimos obter o resultado passando o limite com $i \rightarrow \infty$.

Assim seja $x_{1}>x_{2}$ e note que

$$
U_{i}\left(x_{j}, t\right)=U_{i}\left(x_{j}-\eta_{j},\left[\frac{t}{h_{i}}\right] h_{i}\right) . \quad j=1,2
$$

para algum $\eta_{j}$, onde $0 \leq \eta_{j}<l_{i}$. Assim

$$
\frac{U_{i}\left(x_{1}, t\right)-U_{i}\left(x_{2}, t\right)}{x_{1}-x_{2}} \leq \frac{1}{x_{1}-x_{2}} \sum\left(u_{n}^{2 k}-u_{n-2}^{2 k}\right),
$$

onde $k=\left[t / h_{i}\right]$ e somamos sobre todo $n \in \mathbf{Z}$, com $n \in\left[x_{2}-n_{2}, x_{1}-n_{1}\right]$. Usando o Lema (3.1.2) temos 


$$
\begin{aligned}
\frac{U_{i}\left(x_{1}, t\right)-U_{i}\left(x_{2}, t\right)}{x_{1}-x_{2}} & \leq \frac{1}{x_{1}-x_{2}} \sum\left(u_{n}^{2 k}-u_{n-2}^{2 k}\right) \\
& \leq \frac{E\left(x_{1}-\eta_{1}-x_{2}+\eta_{2}\right)}{\left[t / h_{i}\right] h_{i}\left(x_{1}-x_{2}\right)} \\
& \leq \frac{E\left(x_{1}-\eta_{1}-x_{2}+\eta_{2}\right)}{\left(t-h_{i}\right)\left(x_{1}-x_{2}\right)} \\
& =\frac{E^{\prime}}{t-h_{i}}+\frac{\left(\eta_{2}-\eta_{1}\right) E^{\prime}}{\left(t-h_{i}\right)\left(x_{1}-x_{2}\right)} \\
& \leq \frac{E^{\prime}}{t-h_{i}}+\frac{E^{\prime}}{2\left(t-h_{i}\right)} \leq \frac{3 E^{\prime}}{2\left(t-h_{i}\right)} \\
& <\frac{2 E^{\prime}}{\left(t-h_{i}\right)}=\frac{E}{\left(t-h_{i}\right)}
\end{aligned}
$$

com $\eta_{2}-\eta_{1} \leq l_{i} \leq 1$ para $i$ grande.

Lema 3.1.9 Seja $l_{i} \rightarrow 0$ com $i \rightarrow \infty$ e suponha que para $\phi \in C_{0}^{3}$

$$
\lim _{i \rightarrow \infty} \int_{\infty}^{\infty}\left[U_{i}(x, 0)-u_{0}(x)\right] \phi(x, 0) d x=0
$$

Então u satisfaz (3.3), isto é, u é solução de (3.1) e (3.2).

Prova: Como $\phi$ é limitada, (3.30) acontece se para todo $R>0$,

$$
\lim _{i \rightarrow \infty} \int_{-R}^{R}\left[U_{i}(x, 0)-u_{0}(x)\right] \phi(x, 0) d x=0
$$

Mas como $u_{0}$ é limitada e mensurável existe uma função escada $U_{l}(x, 0)$, constante em $[n l,(n+1) l], n \in \mathbf{Z}$ a qual converge localmente em $L_{1}$ para $u_{0}$, isto é, satisfaz (3.31) Tomamos esta função e definimos o valor inicial da nossa equação diferença (3.6). $u_{n}^{2 k}$ :

Agora a solução $u_{n}^{2 k}$ de (3.6) pode ser escrita na forma abaixo somando e subtraindo

$$
\frac{u_{n}^{2 k+1}-u_{n}^{2 k}}{h}-\frac{u_{n}^{2 k+1}-2 u_{n}^{2 k}+u_{n-1}^{2 k}}{2 l^{2}} \frac{l^{2}}{h}+\frac{f\left(u_{n+1}^{2 k}\right)-f\left(u_{n-1}^{2 k}\right)}{2 l}=0
$$


Multiplicamos (3.32) por $\phi_{n}^{2 k}=\phi(n l, 2 k l)$, somamos e subtraimos por $\phi_{n}^{2 k+1}, \phi_{n+1}^{2 k}$, $\phi_{n-1}^{2 k}$ conseguimos:

$$
\begin{aligned}
\frac{\phi_{n}^{2 k+1} u_{n}^{2 k+1}-\phi_{n}^{2 k} u_{n}^{2 k}}{h} & -u_{n}^{2 k+1} \frac{\phi_{n}^{2 k+1}-\phi_{n}^{2 k}}{h}+\frac{l^{2}}{2 h} \frac{2 \phi_{n}^{2 k}-\phi_{n+1}^{2 k}-\phi_{n-1}^{2 k}}{l^{2}} u_{n}^{2 k} \\
& +\frac{\phi_{n+1}^{2 k} u_{n}^{2 k}-\phi_{n}^{2 k} u_{n-1}^{2 k}}{2 h}+\frac{\phi_{n-1}^{2 k} u_{n}^{2 k}-\phi_{n}^{2 k} u_{n+1}^{2 k}}{2 h} \\
& +\frac{\phi_{n+1}^{2 k} f\left(u_{n+1}^{2 k}\right)-\phi_{n-1}^{2 k} f\left(u_{n-1}^{2 k}\right)}{2 l}-f\left(u_{n+1}^{2 k}\right) \frac{\phi_{n+1}^{2 k}-\phi_{n}^{2 k}}{2 l} \\
& -f\left(u_{n-1}^{2 k}\right) \frac{\phi_{n}^{2 k}-\phi_{n-1}^{2 k}}{2 l}=0
\end{aligned}
$$

Como $\phi$ tem suporte compacto podemos assumir que $\phi_{n}^{2 k}=0$ se $k \geq[T / h]$. Então multiplicamos (3.34) por $h l$ e somamos sobre todo $n \in \mathbf{Z}, R \in \mathbf{Z}_{+}$. Como o primeiro, quarto, quinto e sexto termos são somas telescópias, se cancelam exceto para o primeiro termo com $R=0$. Assim conseguimos

$$
\begin{aligned}
-l \sum_{n} \dot{u}_{n}^{0} \phi_{n}^{0} & +h l\left\{\sum_{R, n}\left[-u_{n}^{2 k+1} \frac{\phi_{n}^{2 k+1}}{h}-\frac{l^{2}}{2 h} \frac{\phi_{n+1}^{2 k}-2 \phi_{n}^{2 k}+\phi_{n-1}^{2 k}}{l^{2}} u_{n}^{2 k}\right]\right. \\
& \left.-\sum_{R, n} f\left(u_{n+1}^{2 k}\right) \frac{\phi_{n+1}^{2 k}-\phi_{n}^{2 k}}{2 l}-\sum_{R, n} f\left(u_{n-1}^{2 k}\right) \frac{\phi_{n}^{2 k}-\phi_{n-1}^{2 k}}{2 l}\right\}=0 .
\end{aligned}
$$

Como $U_{h, l}$ é constante por partes, $\phi$ é suave e as integrais são limites de funções escadas podemos escrever:

$$
\begin{aligned}
-\int_{t=0} U_{h, l} \phi+\delta_{1} & -\iint_{t \geq 0} U_{h, l} \phi_{t}+\delta_{2}-\frac{l^{2}}{2 h}-\iint_{t \geq 0} U_{h, l} \phi_{x x}+\delta_{3} \\
& -\iint_{t \geq 0} f\left(U_{h, l}\right) \phi_{x}+\delta_{4}=0,
\end{aligned}
$$

onde $\delta_{i} \rightarrow 0$ uniformemente $\operatorname{com} h, l \rightarrow 0$. Substituindo $U_{h, l}$ por $U_{i}$, temos:

$$
\begin{aligned}
\iint_{t \geq 0} U_{i} \phi_{t} & +\iint_{t \geq 0} f\left(U_{i}\right) \phi_{x}+\frac{l_{i}^{2}}{2 h_{i}} \iint_{t \geq 0} U_{i} \phi_{x x} \\
& +\int_{t=0} U_{i} \phi=\delta\left(h_{i}, l_{i}\right)
\end{aligned}
$$


onde $\delta\left(h_{i}, l_{i}\right) \rightarrow 0 \operatorname{com} i \rightarrow \infty$.

Façamos $i \rightarrow \infty$. Como $U_{i} \rightarrow u$ localmente em $L_{1}(\operatorname{Lema}(3.1 .6))$ e $l_{i} \rightarrow 0, l_{i}^{2} / h_{i_{-}} \rightarrow 0$, já que $l_{i} / h_{i}$ é limitado, temos:

$$
\iint_{t \geq 0} U_{i} \phi_{t}+\frac{l_{i}^{2}}{2 h_{i}} \iint_{t \geq 0} U_{i} \phi_{x x} \rightarrow \iint_{t \geq 0} u \phi_{t} .
$$

Em seguida de (3.30) temos

$$
\int_{t=0} U_{i} \phi \rightarrow \int_{t=0} u_{0} \phi \quad \operatorname{com} \quad i \rightarrow \infty
$$

Também

$$
\begin{aligned}
\left|\iint_{t \geq 0}\left[f\left(U_{i}\right)-f(u)\right] \phi_{x}\right| & \leq\left\|\phi_{x}\right\|_{L_{\infty}} \iint_{s p t \phi}\left|f\left(U_{i}\right)-f(u)\right| \\
& \leq\left\|\phi_{x}\right\|_{L_{\infty}} \iint_{s p t \phi}\left|f^{\prime}(\xi)\right|\left|U_{i}-u\right|
\end{aligned}
$$

onde $\xi$ é um ponto intermediário entre $U_{i}$ e $u$. Como $\left|U_{i}\right| \leq M$, e $f^{\prime}$ é contínua num compacto, temos que $\left|f^{\prime}\right| \leq L$

Assim existe uma constante $C$ independente de $i$ tal que:

$$
\left|\iint_{t \geq 0}\left[f\left(U_{i}\right)-f(u)\right] \phi_{x}\right| \leq C \iint_{s p t \phi}\left|U_{i}-u\right|
$$

e isto implica de (3.23) e assim

$$
\iint_{t \geq 0} f\left(U_{i}\right) \phi_{x} \rightarrow \iint_{t \geq 0} f(u) \phi_{x} .
$$

Então usando (3.35), (3.36), (3.37) e passando o limite com $i \rightarrow \infty$, temos:

$$
\begin{aligned}
\iint_{t \geq 0} U_{i} \phi_{t} & +\frac{l_{i}^{2}}{2 h_{i}} \iint_{t \geq 0} U_{i} \phi_{x x}+\int_{t=0} U_{i} \phi \\
& \leq \iint_{t \geq 0} f\left(U_{i}\right) \phi_{x} \rightarrow \iint_{t \geq 0} u \phi_{t}+\int_{t=0} u_{0} \phi+\iint_{t \geq 0} f(u) \phi_{x} .
\end{aligned}
$$


Podemos agora completar a prova do teorema (3.1).

O Lema anterior vale para $\phi \in C_{0}^{3}$, mas sabemos que $C_{0}^{3}$ é denso em $C_{0}^{1}$, logo temos que (3.3) acontece para toda $\phi \in C_{0}^{1}$. Assim $u$ é solução do nosso problema, satisfaz (3.4) e (3.5) e $|u| \leq\left\|u_{0}\right\|_{L_{\infty}}$. Com isto provamos a existência de solução da lei de conservação escalar (3.1) com dado inicial (3.2).

\subsection{Unicidade da solução de entropia}

Nesta secção mostraremos que soluções de (3.1) e (3.2) que satisfazem a condição de entropia (3.4) são únicas.

Observação 3.2.1 Chamaremos a solução que satisfaz (3.4) de solução de entropia.

O método da prova que usaremos é uma versão não linear do método de Holmgren. Assim se $u$ e $v$ são soluções de (3.1) e (3.2) mostraremos que $u=v$ quase sempre em $t>0$. Para isto é suficiente mostrar que:

$$
\iint_{t \leq 0}(u-v) \phi=0
$$

para toda $\phi \in C_{0}^{1}$. Agora se $\psi \in C_{0}^{1}$, temos:

$$
\iint_{t \leq 0} u \psi_{t}+f(u) \psi_{x}+\int_{t=0} u_{0} \psi=0
$$

e

$$
\iint_{t \leq 0} v \psi_{t}+f(v) \psi_{x}+\int_{t=0} u_{0} \psi=0
$$

Se subtrairmos estas duas equações obtemos:

$$
\iint_{t \leq 0}(u-v) \psi_{t}+[f(u)-f(v)] \psi_{x}=0
$$

ou

$$
\iint_{t \leq 0}(u-v)\left[\psi_{t}+\frac{f(u)-f(v)}{u-v} \psi_{x}\right]=0
$$


Agora se definirmos $F$ por

$$
F(x, t)=\frac{f(u)-f(v)}{u-v},
$$

então podemos escrever (3.39) como:

$$
\iint_{t \leq 0}(u-v)\left[\psi_{t}+F \psi_{x}\right]=0 .
$$

Se resolvermos a equação linear

$$
\phi_{t}+F \phi_{x}=\phi
$$

para $\phi \in C_{0}^{1}(t>0)$ com $\psi \in C_{0}^{1}$ então (3.40) implicaria em (3.38) e poderiamos concluir que $u=v$ quase sempre.

Observação 3.2.2 F, em geral, não é suave e então não é claro que (3.41) tem uma solução $\psi \in C_{0}^{1}$.

O caminho para contornar esta dificuldade é aproximar $u$ e $v$ (e daí $F$ ), por funções suaves $u_{m}, v_{m}$ e resolvermos a correspondente equação linear:

$$
\psi_{t}^{m}+F_{m} \psi_{x}^{m}=\phi
$$

com coeficiêntes suaves, para $\psi^{m} \in C_{0}^{1}$, onde

$$
F_{m}(x, t)=\frac{f\left(u_{m}\right)-f\left(v_{m}\right)}{u_{m}-v_{m}}
$$

Então

$$
\begin{aligned}
\iint_{t \leq 0}(u-v) \phi & =\iint_{t \leq 0}(u-v)\left[\psi_{t}^{m}+F_{m} \psi_{x}^{m}\right] \\
& =\iint_{t \leq 0}(u-v) \phi_{t}^{m}+\iint_{t \leq 0}(u-v) F_{m} \psi_{x}^{m} \\
& =-\iint_{t \leq 0}(f(u)-f(v)) \phi_{x}^{m}+\iint_{t \leq 0}(u-v) F_{m} \psi_{x}^{m}
\end{aligned}
$$


Logo:

$$
\iint_{t \leq 0}(u-v) \phi=\iint_{t \leq 0}(u-v)\left(F_{m}-F\right) \psi_{x}^{m}
$$

Então se $F_{m} \rightarrow F$ localmente em $L_{1}$ e se $\psi_{x}^{m}$ é limitada, independente de $m$, podemos passar o limite no lado direito da equação e obter:

$$
\begin{aligned}
\iint_{t \leq 0}(u-v) \phi & =\lim _{m \rightarrow \infty} \iint_{t \leq 0}(u-v)\left(F_{m}-F\right) \psi_{x}^{m} \\
& \doteq \iint_{t \leq 0} \lim _{m \rightarrow \infty}(u-v)\left(F_{m}-F\right) \psi_{x}^{m}=0,
\end{aligned}
$$

e portanto (3.38) acontece.

Conseguiremos este resultado abaixo por meio do qual a condição de entropia (3.4) será usada para obter a limitação uniforme sobre $\psi_{x}^{m}$.

Teorema 3.2.1 Seja $f \in C^{2}, f^{\prime \prime}>0$ e sejam u e v soluçóes satisfazendo a condição de entropia (3.4). Então $u=v$ quase sempre em $t>0$.

Prova Para todo $m \in \mathbf{Z}_{+}$, seja

$$
u_{m}=u * w_{m} \quad e \quad v_{m}=v * w_{m},
$$

onde, $w_{m}$ é o núcleo da média usual de raio $1 / m$, isto é, $w_{m}=\frac{1}{m} \eta\left(\frac{x}{m}\right)$ e $*$ denota o produto convolução .

Definimos:

$$
\begin{aligned}
F_{m}(x, t) & =\int_{0}^{1} f^{\prime}\left(\theta u_{m}-(1-\theta) v_{m}\right) d \theta \\
& =\frac{1}{u_{m}-v_{m}} \int_{u_{m}}^{v_{m}} f^{\prime}(s) d s=\frac{f\left(u_{m}\right)-f\left(v_{m}\right)}{u_{m}-v_{m}}
\end{aligned}
$$

e isto resolve (3.42) para $\psi^{m}(x, t)$, sujeito a condição limite $\psi^{m}(x, T)=0$. Seja $T>0$ tal que $\phi=0$ se $t \leq T$. A solução deste problema é dada por

$$
\psi^{m}(x, t)=\int_{T}^{t} \phi\left(x_{m}(s ; x, t), s\right) d s
$$


onde $x_{m}(s ; x, t)$ é a única solução da equação característica

$$
\frac{d x_{m}}{d s}=F_{m}\left(x_{m}, s\right), \quad \text { com } \quad x_{m}(t)=x
$$

Agora

$$
\begin{aligned}
\left|u_{m}(x, t)\right| & =\left|u * w_{m}(x, t)\right|=\left|\int_{\mathbf{R}} u(x-y, t) w_{m}(y) d y\right| \\
& \leq \int_{\mathbf{R}}|u(x-y, t)| w_{m}(y) d y \leq M \int_{\mathbf{R}} w_{m}(y) d y=M
\end{aligned}
$$

e similarmente $\left|v_{m}(x, t)\right| \leq M$. Assim como $f \in C^{2}$ vemos que existe uma constante $M_{1}>0$, independente de $m$, tal que:

$$
\begin{aligned}
\left|F_{m}\right| & \leq \sup _{0 \leq \theta \leq 1}\left|f^{\prime}\left(\theta u_{m}+(1-\theta) v_{m}\right)\right| \\
& \leq \sup _{0 \leq \theta \leq 1}\left|f^{\prime}\left(\theta u_{m}+(1-\theta) v_{m}\right)-f^{\prime}\left(\theta u_{m}\right)+f^{\prime}(\theta) u_{m}\right| \\
& \leq \sup _{0 \leq \theta \leq 1}\left|f^{\prime}\left(\theta u_{m}+(1-\theta) v_{m}\right)-f^{\prime}\left(\theta u_{m}\right)\right|+\left|f^{\prime}\left(\theta u_{m}\right)\right| \\
& \leq \sup _{\xi}\left|f^{\prime \prime}(\xi)\right|+M \leq M+M=M_{1}
\end{aligned}
$$

Assim:

$$
\left|F_{m}(x, t)\right| \leq M_{1}
$$

Esta estimativa nos possibilita mostrar que $\psi^{m} \in C_{0}^{1}(t \geq 0)$. Sabemos que $\psi^{m} \in C^{1}$. Seja $S \subset\{t>0\}$ o suporte compacto da $\phi$. Agora, considere a Figura 3.3. $R$ denota a região em $t \geq 0$ que é limitada pelas duas retas de inclinação $\pm 1 / M_{1}$ e as retas $t=0$ e $t=T$ e $R \supset S$ em seu interior a existência desta região vem do fato de $S$ ser compacto.

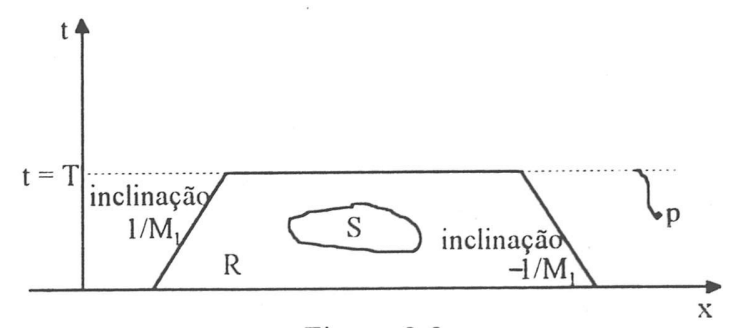

Figura 3.3 
Queremos que spt $\phi \subset R$ para todo $m$. Note que se $t \geq T, \phi=0$ então $\psi^{m}=0$, de (3.44). Alem disso, (3.46) mostra que toda solução de (3.45) que começa no ponto $p$ fora da região $R$ em $t<T$ encontra $t=T$ num ponto que não está em $R$. Segue que $\phi=0$ ao longo de toda esta trajetória.

Assim $\psi^{m}$ satisfaz a equação Homogênea $\psi_{t}^{m}+F_{m} \psi_{x}^{m}=0$ e então $\psi^{m}$ é constante ao longo da trajetória de (3.45).

Como a trajetória encontra $t=T, \psi^{m}=0$ em $t=T$ e daí $\psi^{m}=0$ sobre toda a trajetória, em particular em $p$, logo mostramos que $\psi^{m} \in C_{0}^{1}$ e mais ainda, que $\psi^{m}$ tem suporte no conjunto $R$, independente de $m$.

Segue, como acima, que:

$$
\iint_{t \leq 0}(u-v) \phi=\iint_{t \leq 0}(u-v)\left(F_{m}-F\right) \psi_{x}^{m}
$$

Mostraremos a seguir que $F_{m} \rightarrow F$ localmente em $L_{1}$. Seja $c=\max \left\{f^{\prime \prime}(u):|u| \leq M\right\}$, $c>0$. Então

$$
\begin{aligned}
F(x, t)-F_{m}(x, t) & =\int_{0}^{1}\left[f^{\prime}(\theta u+(1-\theta) v)-f^{\prime}\left(\theta u_{m}+(1-\theta) v_{m}\right)\right] d \theta \\
& =\int_{0}^{1} f^{\prime \prime}(\xi)\left[\theta\left(u-u_{m}\right)+(1-\theta)\left(v-v_{m}\right)\right] d \theta
\end{aligned}
$$

onde $\xi \in\left[\theta u+(1-\theta) v, \theta u_{m}+(1-\theta) v_{m}\right]$, com $|\xi| \leq M$. Assim

$$
\begin{aligned}
\left|F(x, t)-F_{m}(x, t)\right| & \leq c \int_{0}^{1}\left\{\theta\left|u-u_{m}\right|+(1-\theta)\left|v-v_{m}\right|\right\} d \theta \\
& =c\left\{\left|u-u_{m}\right|+\left|v-v_{m}\right|\right\} .
\end{aligned}
$$

Se $K$ é qualquer conjunto compacto em $t \geq 0$ então

$$
\iint_{K}\left|F(x, t)-F_{m}(x, t)\right| \leq c \iint_{K}\left|u-u_{m}\right|+\left|v-v_{m}\right|
$$

onde

$$
\iint_{K}\left|u-u_{m}\right|+\left|v-v_{m}\right| \rightarrow 0 \quad \text { com } \quad m \rightarrow \infty .
$$


Mostraremos agora que podemos limitar $\psi_{x}^{m}$. Primeiro mostraremos para $t \geq \alpha$.

Seja $\alpha>0$ arbitrário então para cada $t \geq \alpha$ fixo, segue da condição de ențropia (3.4) que a função $u(x, t)-E x / \alpha$ é não crescente em $x$, já que se $a>0$,

$$
u(x, t)-\frac{E(x+a)}{\alpha}-u(x, t)+\frac{E x}{\alpha} \leq E a\left(\frac{1}{t}-\frac{1}{\alpha}\right)<0 .
$$

Disso tiramos que

$$
w_{m} *\left(u-\frac{E x}{\alpha}\right)=u_{m}-\frac{E\left(w_{m} * x\right)}{\alpha}
$$

é também não crescente em $x$. Como esta última função é suave e

$$
\frac{\partial}{\partial x}\left[w_{m} *\left(u-\frac{E x}{\alpha}\right)\right]=\frac{\partial u_{m}}{\partial x}-\frac{E}{\alpha},
$$

vemos que os seguintes acontecem:

$$
\frac{\partial u_{m}}{\partial x} \leq \frac{E}{\alpha}, \quad e \quad \frac{\partial v_{m}}{\partial x} \leq \frac{E}{\alpha}
$$

Em seguida, de (3.44) segue

$$
\frac{\partial F_{m}}{\partial x}=\int_{0}^{1} f^{\prime \prime}\left(\theta u_{m}+(1-\theta) v_{m}\right)\left[\theta \frac{\partial u_{m}}{\partial x}+(1-\theta) \frac{\delta v_{m}}{\delta x}\right] d \theta
$$

e como $f^{\prime \prime}>0$, de (3.48) temos que:

$$
\begin{aligned}
\frac{\partial F_{m}}{\partial x} & \leq \int_{0}^{1} f "\left(\theta u_{m}+(1-\theta) v_{m}\right) \frac{E}{\alpha}(\theta+(1-\theta)) d \theta \\
& =\frac{E}{\alpha} \int_{0}^{1} f "\left(\theta u_{m}+(1-\theta) v_{m}\right) d \theta
\end{aligned}
$$

Como f" assume máximo segue

$$
\frac{\partial F_{m}}{\partial x} \leq K_{c k}
$$


onde

$$
K_{\alpha}=\frac{E}{\alpha} \max _{|u| \leq M} f^{\prime \prime}(u)
$$

é independente de $m$.

Seja agora:

$$
a_{m}(s)=\frac{\partial x_{m}}{\partial \bar{x}}(s ; \bar{x}, \bar{t}),
$$

onde $(\bar{x}, \bar{t})$ é um ponto fixo do semi-plano superior. Note que $x_{m}(\bar{t} ; \bar{x}, \bar{t})=\bar{x}$; tal que :

$$
a_{m}(\bar{t})=\frac{\partial x_{m}}{\partial \bar{x}}(\bar{t} ; \bar{x}, \bar{t})=\frac{\partial \bar{x}_{m}}{\partial \bar{x}}=1
$$

Assim,

$$
\begin{aligned}
\frac{\partial a_{m}}{\partial s} & =\frac{\partial}{\partial s} \frac{\partial \bar{x}_{m}}{\partial \bar{x}}=\frac{\partial}{\partial \bar{x}} \frac{\partial x_{m}}{\partial s}=\frac{\partial}{\partial \bar{x}} F_{m}\left(x_{m}, s\right) \\
& =\frac{\partial}{\partial \bar{x}} F_{m}\left(x_{m}(s ; \bar{x}, \bar{t}, s)=\frac{\partial F_{m}}{\partial x} \frac{\partial x_{m}}{\partial \bar{x}}=\frac{\partial F_{m}}{\partial x} a_{m}\right.
\end{aligned}
$$

Então de (3.50) obtemos a fórmula

$$
a_{m}(s)=\exp \left(\int_{\bar{t}}^{s} \frac{\partial F_{m}}{\partial x}\left(x_{m}(\tau), \tau\right) d \tau\right)
$$

Como $\alpha \leq \bar{t} \leq s \leq T$ temos de (3.49):

$$
\begin{aligned}
\left|a_{m}(s)\right| & =a_{m}(s)=e^{\left(\int_{\bar{t}}^{s} \frac{\partial F_{m}}{\partial x}\left(x_{m}(\tau), \tau\right) d \tau\right)} \\
& =e^{\int_{\bar{t}}^{s} K_{\alpha} d \tau}=e^{K_{\alpha}(s-\bar{t})} \leq e^{K_{\alpha}(T-\alpha)}
\end{aligned}
$$

Mas de (3.44)

$$
\frac{\partial \psi^{m}}{\partial x}(x, \bar{t})=\int_{T}^{\bar{t}} \frac{\partial \phi}{\partial x} \frac{\partial x_{m}}{\partial x} d s=\int_{T}^{\bar{t}} \frac{\partial \phi}{\partial x} a_{m} d s
$$


$\log 0$

$$
\left|\frac{\partial \psi^{m}}{\partial x}\right| \leq \int_{T}^{\bar{t}}\left|\frac{\partial \phi}{\partial x}\right|\left|a_{m}\right||d s| \leq \overline{K_{\alpha}} . \quad \text { se } \quad t \geq \alpha
$$

onde $K_{\alpha}$ é independente de $m$.

Agora mostraremos que $\psi^{m}$ é limitada em $0 \leq t \leq \alpha$. Para isto, definimos:

$$
V_{t}\left(\psi^{m}\right)=\int_{-\infty}^{\infty}\left|\frac{\partial \psi^{m}}{\partial x}\right| d x
$$

onde a variação total de $\psi^{m}$ é dada como uma função de $x$, para cada $t>0$. De (3.51) e do fato que as $\psi^{m}$ estão em $C_{0}^{1}$ com suporte contido numa região independente de $m$ temos:

$$
V_{t}\left(\psi^{m}\right) \leq \int_{-\infty}^{\infty} \overline{K_{\alpha}} d x \leq C_{\alpha} \quad \text { se } \quad t \geq \alpha
$$

onde $C_{\alpha}$ não depende de $m$.

A última estimativa que precisamos é

$\exists N \in \mathbf{Z}, \quad$ talque $\quad n>N \Rightarrow V_{t}\left(\psi^{m}\right) \leq C_{1 / n} \quad \forall t, \quad 0<t \leq \frac{1}{n}<\frac{1}{N}$

Para provar isto, note que como $\phi$ tem suporte compacto em $t>0$, existe um $N \in \mathbf{Z}_{+}$tal que, $\phi(x, t)=0$ se $t<1 / N$,

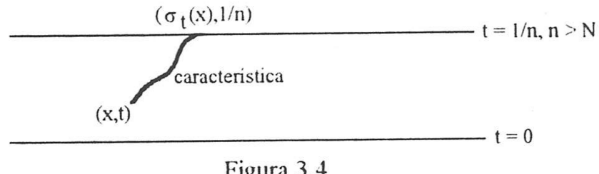
veja Figura 3.4.

Assim de (3.42)

$$
\psi_{t}^{m}+F_{m} \psi_{x}^{m}=0 \quad \text { se } \quad t<\frac{1}{N}
$$

Agora se $n>N$, seja $\sigma_{t}: \mathbb{R} \rightarrow \mathbb{R}$ a bijeção definida para $t<1 / n$ pela solução da equação característica( $(3.45)$, isto é , $\sigma_{t}(x)=x_{m}(1 / n ; x, t)$.

Agora seja $t$ tal que $0<t \leq 1 / n \leq 1 / N$. Então para qualquer sequência finita $x_{1}<x_{2}<\ldots<x_{p}$ temos:

$$
\sum_{k=1}^{p-1}\left|\psi^{m}\left(x_{k+1}, t\right)-\psi^{m}\left(x_{k}, t\right)\right|=\sum_{k=1}^{p-1}\left|\psi^{m}\left(\sigma_{t}\left(x_{k+1}\right), \frac{1}{n}\right)-\psi^{m}\left(\sigma_{t}\left(x_{k}\right), \frac{1}{n}\right)\right|
$$


Isto acontece pois $\psi^{m}$ é constante ao longo das características em vista de (3.54). Assim usando (3.52) para $t=1 / n=\alpha$ segue

$$
\sum_{K=1}^{p-1}\left|\psi^{m}\left(x_{k+1}, t\right)-\psi^{m}\left(x_{k}, t\right)\right| \leq V_{1 / n}\left(\psi^{m}\right) \leq C_{1 / n}
$$

Agora tomando o sup sobre todas as partições em $x$ temos:

$$
V_{t}\left(\psi^{m}\right) \leq C_{1 / n}
$$

e isto prova (3.53).

Podemos agora completar a prova do teorema. Seja $\varepsilon>0$ arbitrário com $N$ definido como em (3.53) escolha $\alpha>0$ tão pequeno que:

$$
\alpha<\frac{1}{n} \leq \frac{1}{N} \quad e \quad 4 M M_{1} C_{1 / n} \alpha<\frac{\varepsilon}{2}
$$

Para este $\alpha$ escolha $M$ grande tal que

$$
\iint_{t \geq \alpha}|u-v|\left|F_{m}-F\right|\left|\psi^{m}\right|<\frac{\varepsilon}{2} \quad \text { se } \quad m \geq M
$$

isto pode ser feito já que $|u-v| \leq 2 M, F_{m} \rightarrow F$ localmente em $L_{1}, \psi^{m} \in C_{0}^{1}(t \geq 0)$ e (3.51) ocorre. Então de (3.47) temos

$$
\left|\iint_{t \geq 0}(u-v) \phi\right| \leq \iint t \geq 0|u-v|\left|F_{m}-F\right|\left|\psi^{m}\right|+\iint t \leq \alpha|u-v|\left|F_{m}-F\right|\left|\psi^{m}\right| .
$$

Agora como $\alpha<1 / n \leq 1 / N$,

$$
\begin{aligned}
\iint_{t \leq \alpha}|u-v|\left|F_{m}-F\right|\left|\psi^{m}\right| & \leq 2 M \cdot 2 M_{1} \iint_{t \leq \alpha}\left|\psi^{m}\right|=4 M M_{1} \int_{0}^{\alpha} \int_{-\infty}^{\infty}\left|\psi^{m}\right| \\
& =4 M M_{1} \int_{0}^{\alpha} V_{t}\left(\psi^{m}\right) d t \\
& \leq 4 M M_{1} C_{1 / n} \cdot \alpha
\end{aligned}
$$


Por (3.55) segue - se

$$
\iint_{t \leq \alpha}|u-v|\left|F_{m}-F\right|\left|\psi^{m}\right| \leq \frac{\varepsilon}{2} .
$$

Assim, usando (3.56) e (3.58) em (3.57) obtemos

$$
\left|\iint_{t \geq 0}(u-v) \phi\right| \leq \varepsilon
$$

e da arbitrariedade de $\varepsilon$

$$
\iint_{t \geq 0}(u-v) \phi=0
$$

para toda $\phi \in C_{0}^{1}(t \geq 0)$. Assim $u=v$ quase sempre em $t>0$. Isto completa a prova da unicidade.

\subsection{Comportamento assintótico das soluções de en- tropia}

Nesta secção estudaremos o comportamento assintótico da solução . Existem dois casos distintos a considerar: O caso onde o dado inicial $u_{0}$ é periódico e o caso onde $u_{0}$ tem suporte compacto.

No caso periódico mostraremos que $u$ tende para o valor médio de $u_{0} \mathrm{com}$ um raio $t^{-1}$, uniformemente em $x$. E no caso em que $u_{0}$ tem suporte compacto $u$ tende uniformemente para zero com $11 \mathrm{~m}$ raio $t^{-1 / 2}$ e tende na norma $L_{1}$ para uma particular função chamada uma $\mathrm{N}$-onda, também com raio $t^{-1 / 2}$.

Observação 3.3.1 Vamos supor que $u$ é diferenciável em alguma região $R$ contida em $0 \leq t \leq T$ e sejam $x_{1}, x_{2}$ duas curvas características em $R$. Já que $u$ é constante ao longo das características, vemos que a variação de $u(., T)$ no intervalo $\left[x_{1}(T), x_{2}(T)\right]$ é igual a variação de $u(., 0)$ no intervalo $\left[x_{1}(0), x_{2}(0)\right]$. 
Observação 3.3.2 Vamos assumir que existe um choque $\gamma$, isto é, uma solução descontínua presente em $u$ entre as duas características $x_{1}$ e $x_{2}$. Veja Figura 3.5.

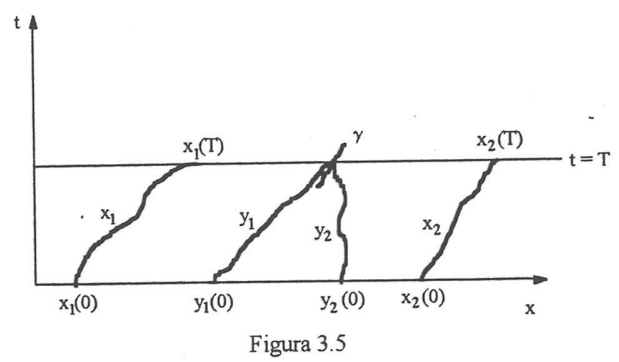

Como $u$ satisfaz a condição de entropia (3.4) sabemos que existem características $y_{1}$ e $y_{2}$ que colidem com o choque em ambos os lados no tempo $t=T$ (veja(3.18)). Segue que

$$
\begin{aligned}
& \operatorname{Var} \cdot \operatorname{Tot}_{\left[x_{1}(T), x_{2}(T)\right]} u(., T)=\operatorname{Var} . T o t_{\left[x_{1}(0), y_{1}(0)\right]} u(., 0)+\operatorname{Var} \cdot \operatorname{Tot}_{\left[y_{2}(0), x_{2}(0)\right]} u(., 0) \\
& <\operatorname{Var} \operatorname{Tot}_{\left[x_{1}(0), x_{2}(0)\right]} u(., 0)
\end{aligned}
$$

Assim na presença de choque a variação total de $u$ entre as características diminui com o tempo. Nosso objetivo é dar uma estimativa qualitativa deste decrescimento.

Começamos considerando o caso onde $u_{0}$ tem suporte compacto, digamos $u_{0}=0$ se $x \in\left[s_{-}, s_{+}\right]$, onde $\left[s_{-}, s_{+}\right]$é o menor intervalo que contém o suporte de $u_{0}$.

Assumimos outra vez que $f$ é convexa; isto é, $f^{\prime \prime}>0$. Por simplicidade assumiremos:

$$
f(0)=f^{\prime}(0)=0
$$

pois podemos substituir $f(u)$ por $f(u)+a u+b$. Também, definimos $k$ e $\mu$ por:

$$
k=f^{\prime}(0) \quad e \quad \mu=\inf _{|u|<M} f^{\prime \prime}(u)>0,
$$

onde $\left\|u_{0}\right\|_{L_{\infty}}=M$. Finalmente definimos duas importantes quantidades:

$$
q=\max _{y} \int_{y}^{\infty} u_{0}(x) d x \quad e \quad-p=\min _{y} \int_{-\infty}^{y} u_{0}(x) d x
$$

Ambos $p$ e $q$ são finitos já que a função

$$
Q(y)=\int_{y}^{\infty} u_{0}(x) d x
$$

é limitada e contínua.

Agora seja $u(x, t)$ a solução única de $(3.1)$ com dado inicial $u_{0}(x)$. Assumimos que 
$u$ é suave por partes (isto é sempre verdade se $f^{\prime \prime}>0$ ) veja [3]. Como $u_{0}$ tem suporte compacto, o mesmo é verdade para $u(., t)$ para cada $t>0$; isto segue de $(3.5)$ fazendo $v_{0}=v=0$. Seja agora

$$
S^{+}(t)=\inf \{y: u(x, t)=0, \forall x>y\}, \quad S^{+}(0)=s_{+} .
$$

e

$$
u^{+}(t)=u\left(S^{+}(t)-0, t\right)=\lim _{\varepsilon \downarrow 0} u\left(S^{+}(t)-\varepsilon, t\right),
$$

e note que $u\left(S^{+}(t)-0, t\right) \equiv 0$. Segue da condição de entropia que $u^{+}(t) \geq 0$ para todo $t>0$ pois

$$
\lim _{\varepsilon \downarrow 0} \frac{u\left(S^{+}(t)-\varepsilon+2 \varepsilon, t\right)-u\left(S^{+}(t)-\varepsilon, t\right)}{2 \varepsilon} \leq \lim _{\varepsilon \downarrow 0} \frac{E}{t}=\frac{E}{t}
$$

assim

$$
\lim _{\varepsilon \downarrow 0} u\left(S^{+}(t)-\varepsilon, t\right)+\frac{2 E \varepsilon}{t} \geq \lim _{\varepsilon \downarrow 0} u\left(S^{+}(t)+\varepsilon, t\right)=0 \quad \forall \varepsilon>0
$$

Portanto

$$
u^{+}(t) \geq 0 \quad \forall t>0 .
$$

Observe que se $u^{+}(t)=0$ então $\left(S^{+}(t), t\right)$ é um ponto de continuidade de $u$.

Caso $S^{+}(t)$ seja diferenciavel, como afirma o autor, como $u^{+}(t) \geq 0$ a inclinação da característica que sai do ponto $\left(S^{+}(t)-0, t\right)$ no tempo para trás é positiva já que $f^{\prime}(0)=0$. Isto mostra que:

$$
\frac{d S^{+}(t)}{d t} \geq 0
$$

Proposição 1 Se $q>0$, então $u^{+}(t) \equiv 0, t>0$ e portanto u é contínua.

Prova Suponhamos $u^{+}(\bar{t})>0$, para algum $\bar{t}>0$. Seja $\mathrm{R}$ a região descrita na Figura 3.6 dada pelas retas $u=S^{+}(t)$, $t=$ const e pela característica $\Gamma_{1}$ Note que a desigualdade de entropia (2.13) mostra que estas características podem ser extendidas para $t=0$ como descrito.

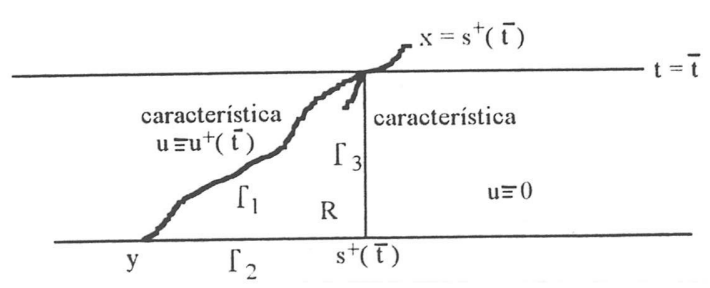

Figura 3.6 
Agora como $u$ é suave por partes, segue do teorema da divergência e de (3.3) que

$$
\int_{\partial \mathrm{R}} u d x-f(u) d t=0
$$

Além disso

$$
\int_{\Gamma_{1}} u d x-f(u) d t=\int_{0}^{\bar{t}}\left(u \frac{d x}{d t}-f\right) d t=\int_{0}^{\bar{t}}\left(u f^{\prime}(u)-f\right) d t
$$

e como $u=u^{+}(\bar{t})$ ao longo de $\Gamma_{1}$ temos:

$$
\int_{\Gamma_{1}} u d x-f(u) d t=\bar{t}\left[\bar{u} f^{\prime}(\bar{u})-f(\bar{u})\right]
$$

onde $\bar{u}=u^{+}(\bar{t})$. Agora

$$
\begin{aligned}
\int_{\Gamma_{2}} u d x-f(u) d t & =-\int_{y}^{S^{+}(\bar{t})} u(x, 0) d x=-\int_{y}^{S^{+}(\bar{t})} u_{0}(x) d x \\
& =-\int_{y}^{\infty} u_{0}(x) d x
\end{aligned}
$$

e

$$
\int_{\Gamma_{3}} u d x-f(u) d t=0
$$

já que $u=0$ em $\Gamma_{3}$ e $f(0)=0$. Usando este resultado em (3.63) temos:

$$
\bar{t}\left[\bar{u} f^{\prime}(\bar{u})-f(\bar{u})\right]=\int_{y}^{\infty} u_{0}(x) d x .
$$

Mas

$$
0=f(0)=f(\bar{u})+f^{\prime}(\bar{u})(0-\bar{u})+\frac{1}{2} f^{\prime \prime}(\xi)(0-\bar{u})^{2},
$$

onde, $0 \leq \xi \leq \bar{u}$. Usando isto em (3.64) segue

$$
o<\frac{1}{2} f^{\prime \prime}(\xi) \bar{u}^{2} \bar{t}=\int_{y}^{\infty} u_{0}(x) d x \leq q
$$


Isto mostra que $q>0$ o que é um absurdo.

Portanto:

$$
u^{+}(t) \equiv 0, \quad t>0
$$

Teorema 3.3.1 Assuma que $f^{\prime \prime}>0, f(0)=f^{\prime}(0)=0$ e que $u_{0}(x)$ é uma função limitada, mensurável tendo suporte compacto. Então se u é a solução de entropia (única) de (3.1), (3.2), u decai para zero com $t \rightarrow \infty$ uniformemente em $x$, com uma razão $t^{-1 / 2}$.

Prova De (3.65) segue que se $u^{+}(t)>0$, então $\mu u^{+}(t)^{2} t / 2 \leq q$, tal que:

$$
u^{+}(t) \leq\left(\frac{2 q}{\mu}\right)^{1 / 2} t^{1 / 2}
$$

Como $\dot{S}^{+}(t)\left(u^{+}(t)-0\right)=f\left(u^{+}(t)\right)-f(0)$ em vista de (3.60) temos

$$
\dot{S}^{+}(t)=\frac{f\left(u^{+}(t)\right)}{u^{+}(t)}=\frac{f^{\prime \prime}(\theta) u^{+}(t)}{2}
$$

onde $0<\theta<u^{+}(t)$. Assim de (3.66), segue

$$
\dot{S}^{+}(t) \leq \frac{c}{2} \sqrt{\frac{2 q}{\mu}} t^{-1 / 2}
$$

onde $c=\max _{|u| \leq M} f^{\prime \prime}(u)$. Logo

$$
S^{+}(t) \leq s_{+}+c \sqrt{\frac{2 q}{\mu}} t^{1 / 2}
$$

Se definirmos

$$
S^{-}(t)=\sup \{y: u(x, t)=0, \forall x<y\}, \quad S^{-}(0)=s_{-}
$$

então um argumento similar nos dá

$$
S^{-}(t) \geq s_{-}-c \sqrt{\frac{2 q}{\mu}} t^{1 / 2}
$$


Seja $\left(x_{ \pm}, t\right)=(x \pm 0, t)$ então como $u$ é constante ao longo das características

$$
u\left(x_{ \pm}, t\right)=u_{0}(y \pm(x, t))
$$

onde do capítulo anterior,

$$
x=y_{ \pm}+t f^{\prime}\left(u\left(x_{ \pm}, t\right)\right)
$$

Como $S^{-}(t) \leq x \leq S^{+}(t)$ e $s_{-} \leq y_{ \pm} \leq s_{+}$temos para $t$ grande

$$
\begin{aligned}
\left|f^{\prime}\left(u\left(x_{ \pm}, t\right)\right)\right| & \leq\left|\frac{x-y_{ \pm}}{t}\right| \\
& \leq \frac{1}{t} \max \left(S^{+}(t)-s_{-}, s_{+}-S^{-}(t)\right) \\
& \leq \text { const } \cdot t^{-1 / 2}
\end{aligned}
$$

Isto segue de (3.69), (3.70) e de desigualdades diferenciais. Agora (3.60) implica que $\left|f^{\prime}(u)\right| \geq \mu|u|$ pois

$$
\begin{gathered}
0=f^{\prime}(0)=f^{\prime}(u)+f^{\prime \prime}(u)(0-u) \Longrightarrow \\
\Longrightarrow\left|f^{\prime}(u)\right|=\left|f^{\prime \prime}(u) u\right| \geq \inf f^{\prime \prime}(u) u=\mu u
\end{gathered}
$$

e assim

$$
|u(x, t)| \leq \text { const } \cdot t^{-1 / 2}
$$

Daqui para frente vamos assumir que $f \in C^{3}$. Podemos agora obter a forma assintótica da solução com $t \rightarrow \infty$. Para este fim observamos que de (3.67) e (3.65) temos:

$$
\dot{S}^{+}(t)=\frac{f^{\prime \prime}(\theta) u^{+}(t)}{2} \leq \frac{1}{2} \frac{f^{\prime \prime}(\theta)}{\sqrt{f^{\prime \prime}}(\xi)} \sqrt{\frac{2 q}{t}}
$$

Quando $t \rightarrow \infty$ segue de (3.71) que $\theta$ e $\xi$ tendem para zero. Assim, como $k=f^{\prime \prime}(0)$,

$$
f \prime(\theta)=k+O(|\theta|) \quad \text { e } \quad f^{\prime \prime}(\xi)=k+O(|\xi|)
$$


Temos, $\theta=O(|u|)=O\left(t^{-1 / 2}\right)$ e $\xi=O\left(t^{-1 / 2}\right)$, em vista de (3.71). Assim,

$$
\frac{f^{\prime \prime}(\theta)}{\sqrt{f^{\prime \prime}(\xi)}}=\sqrt{k} \frac{1+O\left(t^{-1 / 2}\right)}{\sqrt{1+O\left(t^{-1 / 2}\right)}}=\sqrt{k}\left(1+O\left(t^{-1 / 2}\right)\right)
$$

e disto segue que:

$$
\dot{S}^{+} \leq \sqrt{\frac{k q}{2}} t^{-1 / 2}+O\left(t^{-1}\right)
$$

logo,

$$
S^{+}(t) \leq s_{+}+\sqrt{2 k q} t^{1 / 2}+O(\ln (t))=s_{+}+\left[\sqrt{2 k q}+O\left(t^{-1 / 2} \ln t\right)\right] t^{1 / 2}
$$

Similarmente:

$$
S^{-}(t) \geq s_{-}-\left[\sqrt{2 k q}+O\left(t^{-1 / 2} \ln t\right)\right] t^{1 / 2}
$$

Agora definimos as funções $w(x, t)$ e $\tilde{w}(x, t)$ por:

$$
w(x, t)= \begin{cases}x / k t & \text { se } s_{-}-\sqrt{2 k p} t^{1 / 2}<x<s_{+}+\sqrt{2 k p} t^{1 / 2} \\ 0 & \text { c.c. }\end{cases}
$$

Veja Figura 3.7.

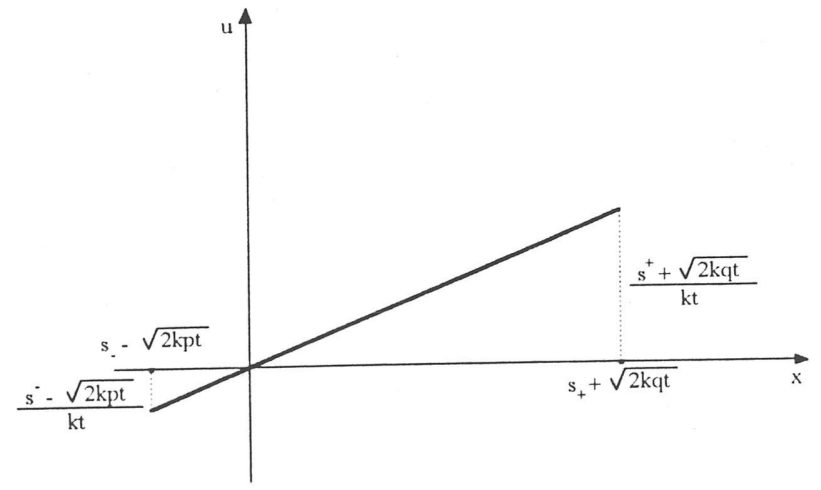

Figura 3.7

$\tilde{w}(x, t)= \begin{cases}x / k t & \text { se } s_{-}-\left[\sqrt{2 k q}+O\left(t^{-1 / 2} \ln t\right)\right] t^{1 / 2}<x<s_{+}+\left[\sqrt{2 k q}+O\left(t^{-1 / 2} \ln t\right)\right] t^{1 / 2}, \\ 0 & \text { c.c. }\end{cases}$ 
Então como $u(x, t)=u_{0}(y(x, t))$, onde $s_{-}<y(x, t)<s_{+}$, e $t f^{\prime}(u(x, t))=x-y(x, t)$ e usando Taylor em $\theta$, temos $f^{\prime}(\theta)=f^{\prime}(0)+f^{\prime \prime}(0)(\theta-0)+O\left(|\theta|^{2}\right)$. Substituindo $\theta=u(x, t)$ segue

$$
f^{\prime \prime}(0) u(x, t)+O\left(|u|^{2}\right)=\frac{x-y(x, t)}{t}
$$

Como $k=f^{\prime \prime}(0)$ então

$$
u(x, t)=\frac{x}{k t}-\frac{y(x, t)}{k t}+O\left(|u|^{2}\right)=\frac{x}{k t}+O\left(t^{-1}\right)
$$

usando (3.71). Consequentemente, para $t$ grande,

$$
\begin{aligned}
\int_{-\infty}^{\infty}|u(x, t)-\tilde{w}(x, t)| d x & =\int_{s_{-}-\sqrt{2 k p} t^{1 / 2}+O(\ln t)}^{s_{+}+\sqrt{2 k p} t^{1 / 2}+O(\ln t)}\left|u(x, t)-\frac{x}{k t}\right| d x \\
& \leq \int_{s_{-}-\sqrt{2 k p} t^{1 / 2}+O(\ln t)}^{s_{+}+\sqrt{2 k p} t^{1 / 2}+O(\ln t)} O\left(t^{-1}\right) d x \leq \frac{M}{t} \int_{s_{-}-\sqrt{2 k p} t^{1 / 2}+O(\ln t)}^{s_{+}+\sqrt{2 k p} t^{1 / 2}+O(\ln t)} d x \\
& \leq \operatorname{const} \cdot t^{-1}\left[1+t^{1 / 2}+\ln t\right] \leq \text { const } \cdot t^{1 / 2}
\end{aligned}
$$

aqui, usamos (3.75). Isto mostra que:

$$
\|u(., t)-\tilde{w}(., t)\|_{L_{1}(\mathbf{R})}=O\left(t^{-1 / 2}\right) \quad \operatorname{com} \quad t \rightarrow \infty
$$

Similarmente,

$$
\|w(., t)-\tilde{w}(., t)\|_{L_{1}(\mathbf{R})}=O\left(t^{-1 / 2}\right) \quad \text { com } \quad t \rightarrow \infty
$$

Podemos assim concluir que:

$$
\|u(., t)-w(., t)\|_{L_{1}(\mathbf{R})}=O\left(t^{-1 / 2}\right) \quad \text { com } \quad t \rightarrow \infty
$$

A função $w(x, t)$, definida por (3.74) é chamada uma "N-onda", por causa do seu perfil em cada $t>0$. Temos assim mostrado que a condição de entropia decai para uma "Nonda" em $L_{1}(\mathbb{R})$ com $t \rightarrow \infty$ contanto que as hipóteses do Teorema (3.3.1) ocorrem.

Voltamos nossa atenção agora para o caso onde $u_{0}$ é uma função periódica, digamos de período $p$. Assumimos por simplicidade, que $u_{0}$ é também monótona por partes. Temos então o seguinte teorema. 
Teorema 3.3.2 Seja $f^{\prime \prime}>0$, e seja $u_{0} \in L_{\infty}(\mathbb{R})$ uma função monotônica por partes, periódica de período p. Seja u a solução de (3.1) e (3.2) a qual satisfaz a condição de entropia (3.4). Então:

$$
\left|u(x, t)-\bar{u}_{0}\right| \leq \frac{2 p}{h t}
$$

onde, $\bar{u}_{0}=\frac{1}{p} \int_{0}^{p} u_{0}(x) d x$, e $h=\min \left\{f^{\prime \prime}(u):|u| \leq\left\|u_{0}\right\|_{L_{\infty}}\right\} . \quad$ Assim, u decai para o valor médio de $u_{0}$ com razão $t^{-1}$, esta razão é maior que o caso onde $u_{0}$ tem suporte compacto, veja(3.71).

Antes de provarmos este teorema, temos o seguinte lema.

Lema 3.3.1 Se $u_{0}$ satisfaz as hipóteses do teorema anterior e u é a solução única de entropia de (3.1) e (3.2) então para cada $t>0, u(., t)$ é periódica de período $p$.

Prova: Seja $v(x, t)=u(x+p, t)$. Então para $\phi \in C_{0}^{1}(t>0)$,

$$
\begin{aligned}
\iint_{t>0} v \phi_{t}+f(v) \phi_{x}+\int_{t=0} u_{0} \phi & =\iint_{t>0} u(x+p, t) \phi_{t}+f(u(x+p, t)) \phi_{x}+\int_{t=0} u_{0} \phi \\
& \left.=\iint_{t>0} u(y, t) \phi_{t}+f(u(y, t))\right) \phi_{x}+\int_{t=0} u_{0} \phi=0
\end{aligned}
$$

onde $y=x+p$. Assim v é solução de (3.1) e (3.2). Como $v$ satisfaz a condição de entropia (3.4), temos que $v(x, 0)=u_{0}(x)$ e pela unicidade do teorema $(3.2), v(x, t)=u(x, t)$. $u(., t)$ é p-periódica.

Vamos agora provar o teorema (3.3.2)

Prova Sejam $x_{1}(t)$ e $x_{2}(t)$ duas características quaisquer de (3.1). Então $x_{i}, i=1,2$ satifazem a equação diferencial ordinária

$$
\frac{d x}{d t}=f^{\prime}(u(x, t))
$$

Defina a "largura" $D(t)$ da faixa $0 \leq t \leq T$ limitada pelas duas características, como

$$
D(t)=x_{2}(t)-x_{1}(t)
$$


Então,

$$
D^{\prime}(t)=x_{1}^{\prime}(t)-x_{2}^{\prime}(t)=f^{\prime}\left(u_{2}\right)-f^{\prime}\left(u_{1}\right)
$$

onde $u_{i}=u_{i}\left(x_{i}(t), t\right)$ são constantes, $i=1,2$. Como $f^{\prime \prime} \geq h>0, u_{2}>u_{1}$ implica que $f^{\prime}\left(u_{2}\right)-f^{\prime}\left(u_{1}\right)>h\left(u_{2}-u_{1}\right)$, temos que:

$$
D^{\prime}(t) \leq h\left(u_{2}-u_{1}\right)
$$

se integrarmos com respeito a $t$ encontramos;

$$
\begin{gathered}
\int_{0}^{T} D^{\prime}(t) d t \leq \int_{0}^{T} h\left(u_{2}-u_{1}\right) d t \\
\Longrightarrow D(T)-D(0) \leq h\left(u_{2}-u_{1}\right) \int_{0}^{T} d t \\
\Longrightarrow D(T) \leq D(0)+h\left(u_{2}-u_{1}\right) T, \quad u_{2}>u_{1} .
\end{gathered}
$$

Seja $I$ um intervalo no eixo $x$. Como $u_{0}$ é monótona por partes, podemos dividir $I$ em subintervalos $y_{0}<y_{1}<\cdots<y_{n}$ tal que $u_{0}$ é alternadamente crescente e decrescente no subintervalo $\left[y_{i-1}, y_{i}\right]$. Seja $y_{i}$ a característica gerada por $y_{i}$, isto é,

$$
\frac{d y_{i}}{d t}=f^{\prime}\left(u\left(y_{i}, t\right)\right), \quad y_{i}(0)=y_{i}
$$

Convencionaremos que se $y_{i}(t)$ encontra um choque, $y_{i}$ continua sendo um choque. Agora podemos ver que para cada $t>0, u(x, t)$ é alternadamente crescente e decrescente no intervalo $\left(y_{i-1}(t), y_{i}(t)\right)$.

Seja $I(t)$ o intervalo $\left[y_{0}(t), y_{n}(t)\right]$, e seja $L(t)$ seu comprimento. Definimos:

$$
V_{+}(t)=\text { variação total crescente de } u(., t) \text { sobre } I(t) \text {. }
$$

Faremos a convenção que se $u(., t)$ é crescente em $\left(y_{i-1}, y_{i}\right)$, se e somente se, $i$ é ímpar.

Agora para $i$ ímpar, seja:

$u_{i-1}(t)=$ valor de $u$ na extremidade direita de $y_{i-1}(t)$,

$u_{i}(t)=$ valor de $u$ na extremidade esquerda de $y_{i}(t)$.

Veja Figura 3.8

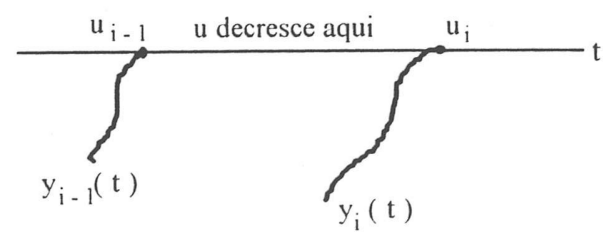

Figura 3.8 
Como a condição de entropia (3.4) implica que $u$ decresce através do choque temos:

$$
V_{+}(t)=\sum_{i_{\text {impar }}}\left[u_{i}(t)-u_{i-1}(t)\right]
$$

Se $y_{i}(t)=y_{i-1}(t)$ então as características vem juntas no ponto $\left(y_{i}(t), t\right)$ tal que este ponto mora no choque, e não temos o i-ésimo termo em (3.78). Por outro lado, se $y_{i-1}(t)<y_{i}(t)$ então existem características $\chi_{i-1}$ e $\chi_{i}$ começando em $t=0$ em $\left[y_{i-1}, y_{i}\right]$ a qual, no ponto $t$, percorre $y_{i-1}(t)$ e $y_{i}(t)$, respectivamente (naturalmente, $\chi_{j}(t)$ será igual $y_{j}(t)$ se $y_{j}(t)$ não continua com o choque). Se colocarmos,

$$
D_{i}(t)=\chi_{i}(t)-\chi_{i-1}(t)
$$

temos que,

$$
D_{i}(t) \geq D_{i}(0)+h\left(u_{i}-u_{i-1}\right) t
$$

Se $i$ é ímpar então

$$
\begin{aligned}
\sum_{i \text { impar }} D_{i}(t) & \geq \sum_{i \text { impar }} D_{i}(0)+h t \sum_{i \text { impar }}\left(u_{i}-u_{i-1}\right) \\
& =\sum_{i \text { impar }} D_{i}(0)+h t V_{+}(t) .
\end{aligned}
$$

Como os intervalos $\left[\chi_{i-1}(t), \chi_{i}(t)\right]$ são disjuntos e moram em $I(t)$, seu comprimento total quando muito é $L(t)$. Assim:

$$
L(t) \geq h t V_{+}(t)
$$

$\mathrm{e}$

$$
V_{+}(t) \leq \frac{L(t)}{h t}
$$

Do Lema (3.3.1) u(.,t) é periódica de período $p$. Se $L(0)=p$ então $L(t)=p$ já que $y_{0}(t) \equiv y_{n}(t)$. Então de (3.79) obtemos:

$$
V_{+}(t) \leq \frac{p}{h t}
$$


Como a variação total crescente de uma função periódica no período é igual a metade da variação total temos:

$$
V(t) \leq \frac{2 p}{h t}, \quad t>0
$$

Onde $V(t)$ denota a variação total de $u$ sobre um período.

Agora seja $\Gamma$ o contorno descrito na Figura 3.9.

Então de (3.63), temos:

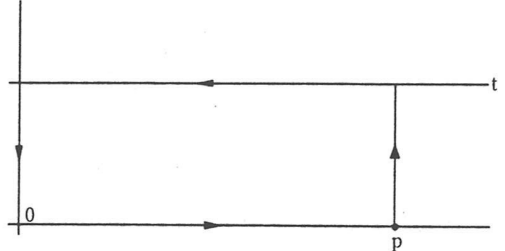

Figura 3.9

$$
0=\int_{0}^{p} u_{0}(x) d x-\int_{0}^{t} f(u(p, \tau)) d \tau+\int_{p}^{0} u(x, t) d x-\int_{t}^{0} f(u(0, \tau)) d \tau
$$

Como $u$ é periódica de período $p$, temos:

$$
\int_{0}^{p} u(x, t) d x=\int_{0}^{p} u_{0}(x) d x
$$

Assim o valor médio de $u$ é independente de $t \mathrm{e}$

$$
\begin{aligned}
\left|u(x, t)-\bar{u}_{0}\right| & =\left|u(x, t)-\frac{1}{p} \int_{0}^{p} u(z, t) d z\right| \\
& =\left|\frac{1}{p} \int_{0}^{p} u(x, t)-u(z, t) d z\right| \\
& \leq \frac{1}{p} \int_{0}^{p}|u(x, t)-u(z, t)| d z \\
& \leq \frac{1}{p} \int_{0}^{p} V(t) d z \\
& =V(t)
\end{aligned}
$$

Logo

$$
\left|u(x, t)-\bar{u}_{0}\right|=V(t) \leq \frac{2 p}{h t}
$$




\section{Bibliografia}

[1] J.Smoller., Shock Waves and Reaction-Diffusion Equation, Springer-Verlag, New York, Heidelberg Berlin, 1982.

[2] I. P. Natanson, Theory of Functions of a Real Variable, Frederick Ungar Publishing Co., United States of America, 1955.

[3] R. J. DiPerma, Singularities of Solutions os Nonlinear Hyperbolic Systems of Conservation Laws, Arch. Rat. Mech. Anal., 60 (1975), pp. 75-100.

[4] P. D. Lax, Hyperbolic Systems of Conservation Laws, Comm. Pure Appl. Math., 10 (1957), pp. 537-566.

[5] P. D. LAX, Weak Solutions of nonlinear Hiperbolic equations and their numerical computations, Comm. Pure Appl. Math., 7 (1954), pp. 159-193.

[6] E. D. Conway, The Formation and Decay of Shocks for a Conservation Laws in Several Dimensions, Arch. Rat. Mech. Anal., 64 (1977), pp. 47-57.

[7] O. Olienik, Discontinuous solutions of nonlinear differential equations, Usp. Mat. Nauk. (N.S), 12 (1957), pp. 3-73, English transl. in Amer. Math. Soc. Transl. Ser. 2, 26, pp. 95-172. 